\title{
Architecture and permeability of post-cytokinesis plasmodesmata lacking
} cytoplasmic sleeves

William J. Nicolas ${ }^{1}$, Magali S. Grison ${ }^{1}$, Sylvain Trépout ${ }^{2}$, Amélia Gaston ${ }^{1,3}$, Mathieu Fouché1,3, Fabrice P. Cordelières ${ }^{4}$, Karl Oparka 5 , Jens Tilsner6,7, Lysiane Brocard ${ }^{8 *}$, and Emmanuelle M. Bayer ${ }^{1 *}$

1. Laboratory of Membrane Biogenesis, UMR5200 CNRS, University of Bordeaux, 71 Avenue Edouard Bourlaux, 33883 Villenave d'Ornon cedex, France

2. Institut Curie, Centre de Recherche, Bât. 112, Centre Universitaire, 91405 Orsay Cedex, France

3. Present address: UMR 1332 BFP, INRA, Univ. Bordeaux, Villenave d'Ornon 33140, France

4. Bordeaux Imaging Centre, UMS 3420 CNRS, CNRS-INSERM-University of Bordeaux 146, rue Léo Saignat, 33076 Bordeaux

5. Institute of Molecular Plant Sciences, University of Edinburgh, Edinburgh EH9 3BF, United Kingdom

6. Biomedical Sciences Research Complex, University of St Andrews, Fife KY16 9ST, United Kingdom

7. Cell and Molecular Sciences, The James Hutton Institute, Dundee DD2 5DA, United Kingdom

8. Bordeaux Imaging Centre, Plant Imaging Plateform, UMS 3420, INRA-CNRS-INSERMUniversity of Bordeaux, 71 Avenue Edouard Bourlaux, 33883 Villenave-d'Ornon Cedex, France

* Correspondence should be addressed to:

emmanuelle.bayer@u-bordeaux.fr; Phone: +33 (0) 557122539

lbrocard@bordeaux.inra.fr; Phone ++33 (0) 557122551

Running title: plasmodesmata structure by electron tomography

\section{AbSTRACT}


34 Plasmodesmata are remarkable cellular machines responsible for the controlled 35 exchange of proteins, small RNAs and signalling molecules between cells. They are lined 36 by the plasma membrane (PM), contain a strand of tubular endoplasmic reticulum (ER), 37 and the space between these two membranes is thought to control plasmodesmata 38 permeability.

39 Here, we have reconstructed plasmodesmata 3D ultrastructure with an unprecedented 40 level of 3D information using electron tomography. We show that within 41 plasmodesmata, ER-PM contact sites undergo substantial remodelling events during cell 42 differentiation. Instead of being open pores, post-cytokinesis plasmodesmata present 43 such intimate ER-PM contact along the entire length of the pores, that no inter44 membrane gap is visible. Later on, during cell expansion, the plasmodesmata pore 45 widens and the two membranes separate, leaving a cytosolic sleeve spanned by tethers 46 whose presence correlates with the appearance of the intermembrane gap. Surprisingly, 47 the post-cytokinesis plasmodesmata allow diffusion of macromolecules despite the 48 apparent lack of an open cytoplasmic sleeve, forcing the reassessment of the 49 mechanisms that control plant cell-cell communication. 


\section{INTRODUCTION}

Plasmodesmata are membrane-lined channels that cross the plant cell wall and allow the exchange of molecules between virtually all plant cells. Plasmodesmata are required for coordinated plant growth and development, plant defence signalling $1-11$ and are also exploited by viruses to spread from cell-to-cell and systemically throughout the plant 12,13 .

Plasmodesmata are characterised by the apposition of two membranes: the plasmamembrane (PM) which lines the plasmodesmal pore and a strand of tubular endoplasmic reticulum (ER), which is tightly constricted into a rod-like structure known as the desmotubule ${ }^{14-16}$. These two plasmodesmal membrane compartments are highly specialised and contain specific sets of proteins and lipids, both of which are critical for proper function ${ }^{7,8,17-22}$. Inherent to their structure, plasmodesmata constitute a specialised type of membrane contact site (MCS), a general term describing areas of close (10-30 nm) apposition between two membranes ${ }^{23-25}$. In yeast and human cells MCS are well established sites for inter-organelle signalling, non-vesicular lipid exchange and calcium homeostasis ${ }^{26-29}$. In plasmodesmata, the function of ER-PM contacts remains an enigma ${ }^{30}$ and signalling between the two membranes is still speculative. In current models however, the gap between the two membranes, the cytoplasmic sleeve, defines the space available for molecular trafficking, governing the size exclusion limit (SEL) of the pores. Plasmodesmata symplastic connectivity is strongly regulated in space and time. Their SEL can be modulated in response to biotic/abiotic stresses but also varies depending on the cell type and stage of tissue differentiation ${ }^{7-9,31-36}$. In any cases, the structural plasticity of plasmodesmata is assumed to be critical to adjust symplastic connectivity through the regulation of ER-PM spacing2,36,37. However, how plasmodesmata channels are built and organised within the narrow space between the ER and the PM, and how ER-PM spacing affects cell-to-cell connectivity remains little understood. While spectacular advances have been made over the last decade in imaging supramolecular structures such as the nuclear pore complex ${ }^{38}$, we currently have no data on the 3D structure of plasmodesmata in higher plants. Past studies have greatly contributed to models of plasmodesmata ultrastructure ${ }^{14,39,40}$ but they were based on 2D transmission electron micrographs where no depth (z-axis) and no information on the true 3D organisation of membrane 


\section{1}

contacts within plasmodesmata was available. Yet, in depth understanding of plasmodesmata architecture and how it relates to intercellular connectivity is critical to understand their mechanisms of action.

Here we used electron tomography to gain access to the ultrastructure of plasmodesmata with an unprecedented level of 3D information and shed light on the structural plasticity of their ER-PM junctions. By acquiring multiple snapshots of plasmodesmata at different stages we reconstructed the structural dynamics of their architecture from their biogenesis to later maturation events. We unexpectedly show that within the plasmodesmal pores, ER-PM contacts undergo extensive remodelling, which varies from very tight contacts to intermembrane gaps of about $10 \mathrm{~nm}$, spanned by spokes. Differences in ER-PM connections set apart two plasmodesmata morphotypes, which occurrence correlates with tissue growth and differentiation. Type II display archetypal organisation, with a cytoplasmic sleeve spanned by spoke-elements, and correspond to "mature" plasmodesmata. Contrary to the textbook model, we show that these "spokes" are insensitive to F-actin polymerization inhibitor drugs, suggesting they may not be related to the cytoskeleton. In addition to the archetypal plasmodesmata, we observed a second morphotype (Type I), which occurs in postcytokinesis walls and unexpectedly presents such a tight contact between the ER and the PM that no visible intermembrane space remains. Despite the lack of visible cytoplasmic sleeve, these plasmodesmata are surprisingly capable of non-targeted movement of macromolecules such as GFP. Transition from Type I to Type II plasmodesmata is correlated with cell differentiation and tissue growth. Based on our data we propose that membrane-tethering elements control plasmodesmata MCS maturation and define different functional states of the plasmodesmata channels.

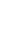
9

(1) 
127 ER-PM spacing within plasmodesmata is regulated during tissue differentiation in 128 root tips.

129 To analyse the ultrastructural organisation of plasmodesmata during tissue 130 differentiation, we first focused on Arabidopsis root tip columella (COL), a tissue 131 involved in gravitropism and soil excavation ${ }^{41-43}$. COL cells offered excellent 132 plasmodesmata preservation after cryo-fixation and freeze-subtitution but also 133 unequivocal traceability of cell lineage where plasmodesmata modification from early 134 formation to later maturation stages can be easily traced. The columella is organised 135 into several cell layers (Fig. 1a); the mitotically active COL cell initials (CCI), situated 136 immediately below the root quiescent center (QC), which divide periclinally providing 137 primary plasmodesmata on their division walls and supplying new COL cell layers ${ }^{44}$ 138 (identified as $\mathrm{C} 1$ for the inner-most layer, followed by C2, C3 etc. where the outer-most 139 layer (CO) ultimately sloughs off ${ }^{45}$ ). Unlike the CCI, the COL cells from C1 to CO are 140 unable to divide and undergo drastic cell elongation. This tissue therefore offers an 141 excellent framework to track down potential modification in plasmodesmata 142 architecture during cell differentiation.

143 Root tissues from one-week-old seedlings were high-pressure-frozen and freeze 144 substituted to stay as close as possible to native conditions, then processed for electron 145 tomography. The outermost cell layers of the COL (from C2 to C4) featured 146 plasmodesmata with an archetypal ultrastructural organisation ( $\mathrm{n}=15$; Fig. 1d). All 147 presented a central desmotubule visible as an electron-opaque rod and an electron148 lucent cytoplasmic sleeve spanned by multiple spoke-like tethers connecting the 149 desmotubule to the PM (Fig. 1d, yellow arrows). These plasmodesmata had an average diameter (PM-PM; inner leaflets) of $39.3 \pm 9.8 \mathrm{~nm}$, while the desmotubule had a 151 diameter of $19.15 \pm 2.5 \mathrm{~nm}$, and they traversed thick cell walls (Fig. 1e,f) and could 152 display branched morphology (data not shown). On the opposite boundary, at the CCI 153 interfaces, newly established plasmodesmata were of drastically different appearance. 154 They presented a grainy electron dense interior with surprisingly no detailed internal 155 features and no visible cytoplasmic sleeve ( $n=20$; Fig. 1b). They traversed the very thin 156 CCI walls (79.1 $\pm 44.5 \mathrm{~nm}$; Fig. 1e) and their average diameter (PM-PM; inner leaflets) 157 was significantly smaller $(23.2 \pm 5.4 \mathrm{~nm})$ than plasmodesmata encountered in 
158 differentiated COL cells (Fig. 1f). Although not readily visible, nearly all plasmodesmata 159 observed ( $\mathrm{n}=18$ out of 20 ) presented cortical ER entering the pores in the form of an 160 electron-dense rod suggesting that despite their tiny size they contained a desmotubule

161 (Fig. 1b, black arrowheads). When comparing the size of the desmotubule in archetypal 162 COL plasmodesmata, to the diameter of the CCI pores, we found no significant difference 163 (Fig. 1f), suggesting that these non-canonical plasmodesmata presented an 164 unconventional and underrated organisation with a very close ER-PM apposition within 165 the entire length of the pores. For clarity, we called plasmodesmata with no visible 166 cytoplasmic sleeve Type I (CCI) and Type II when both the ER-PM electron-lucent gap 167 and the spoke elements were present (from C2 to C4). A transitional stage, between 168 Type I and Type II, was visible at the C1 cell layer (Fig. 1c). While Type I plasmodesmata predominate at the $\mathrm{CCI} / \mathrm{C} 1$ cell interface, the opposite transverse cell wall (the C1-C2 interface) displayed plasmodesmata with intermediate structural organisation and a

171 partially "opened" cytoplasmic sleeve $(n=15)$. At this stage however, spoke-like tethers 172 were difficult to distinguish.

173 Altogether our results indicate that ER-PM contacts within plasmodesmata may be 174 differentially regulated during tissue differentiation in COL cells and reveal unexpected 175 ultrastructural organisation of plasmodesmata in the CCI where no visible electron176 lucent cytoplasmic sleeve remains between the two tightly apposed membranes.

Type II plasmodesmata membrane tethers control ER-PM gaps and are actinindependent.

The archetypal Type II plasmodesmata are characterised by the presence of 181 spoke-like tethers that appeared as fine, electron-dense strands bridging the membranes across the cytoplasmic sleeve (Fig. 2a-c). In COL cells, the spokes were numerous and regularly spaced, with an average of $8 \pm 3 \mathrm{~nm}(\mathrm{n}=8$ plasmodesmata tomograms, 112 tethering elements measured) between the tethers and often the position of the tethers on opposite sides of the desmotubule matched up (Fig. 2a,b yellow arrow). Their length varied from $4 \mathrm{~nm}$ to $20 \mathrm{~nm}$ with a mean value of $9.7 \pm 3.3$ nm (Fig. 2d). Most tethers consisted of single, unbranched filaments, but V- and Yshaped tethers were occasionally observed, in which case the two branches could either connect to the PM or to the desmotubule (Fig. 2e). Spokes of similar appearance and 
length were also observed in Arabidopsis cultured cell plasmodesmata (Fig.2d; see also 191 Fig. 3b).

192 The spoke appearance was concomitant with the transition from Type I to Type II, 193 suggesting that these structures may be involved in controlling the spacing between the 194 desmotubule and the PM. In root COL cells but also in Arabidopsis cultured cells, 195 transitioning or Type II plasmodesmata sometimes presented subsections of the 196 cytoplasmic sleeve gap devoid of spokes (Suppl. Fig. 1 and Fig. 3b) that seemed larger 197 and looser when compared to cytoplasmic sleeve gaps spanned by spokes. To quantify 198 this difference, we measured the cytoplasmic sleeve gaps in subsections of Type II pores 199 harbouring cytoplasmic sleeve gaps spanned by or devoid of spoke-like tethers. The 200 distance between the two membranes was maintained at around $9.6 \pm 2.44 \mathrm{~nm}$ when 201 the spokes were present whereas this distance nearly doubled, and was more variable, 202 when the spokes were absent $18.38 \pm 7.26 \mathrm{~nm}$ (Fig. 2f; see also similar results for 203 cultured cells). Our data therefore suggests that there is a direct correlation between the presence of spoke-like tethers and the control of ER-PM spacing.

In conventional models of plasmodesmata, the spokes are often depicted as 206 myosin molecules that would tether the two membranes by binding with F-actin 207 imbedded in the desmotubule 46-51. This model was built upon immunological data ${ }^{52-54}$ 208 and functional cell-to-cell communication tests ${ }^{49,55}$. If true, disturbing the delicate 209 synthesis/degradation balance of F-actin homeostasis could affect plasmodesmata 210 structure and more especially the spokes elements. In this regard, Arabidopsis roots 211 were treated with drugs altering F-actin polymerization (Latrunculin B and Cytochalasin 212 D) and processed for tomography. Efficiency of the treatments was checked using the 213 actin markers fimbrin actin binding domain 2-green fluorescent protein (35S::GFP214 fABD2-GFP) ${ }^{56}$. We also used the Golgi markers, N-acetylglucosamine1 (NAG1) ${ }^{57}$ and the 215 SNARE protein MEMBRIN12 (MEMB12) ${ }^{58}$ to test their mobility after drug treatment as 216 the cytosolic streaming of Golgi vesicles is known to heavily rely on the actin network ${ }^{59}$ 217 (Supplementary movie 2). Despite alteration of the actin filament network (Fig. 2g-i), 218 the spokes were still observed spanning the cytoplasmic sleeve (Fig. 2j,k) and we saw no 219 significant differences in the pore dimensions or in the length distribution of the spoke220 elements (Fig. 2d,l).

221 We therefore concluded that the spokes may control ER-PM spacing within the 222 plasmodesmata pores but their nature remains unclear. The fact that treatments with F- 
223 actin polymerization inhibitors did not affect plasmodesmal ultrastructure suggests the

224 spokes could be stable actin elements or not cytoskeleton related ${ }^{15}$.

Post-cytokinesis plasmodesmata display very close appositions between the desmotubule and the PM

A surprising outcome of Type I plasmodesmata organisation in the root CCI was the absence of a visible cytoplasmic sleeve. These plasmodesmata were only present in the mitotically active CCI and transition to Type II occurred rapidly across a single cell interface. To get more information about these non-canonical plasmodesmata, we next turned to Arabidopsis liquid cultured cells which are actively dividing, contain primary plasmodesmata ${ }^{60}$ (Suppl. Fig. 3) and do not undergo cell differentiation.

An initial survey revealed the presence of both Type I and II plasmodesmata (Fig. 3a-b). Similar to the CCI, Type I plasmodesmata had a grainy appearance as if the pores were filled with electron dense material throughout the entire channel, with no cytoplasmic sleeve visible nor apparent sub-elements (Fig. 3a). Close examination of tomograms revealed the presence of ER membranes entering the pores (15 out of 17), supporting the presence of a desmotubule (Fig. 3c, yellow arrow; Supplementary movie 4). Type II plasmodesmata in cultured cells displayed a visible cytoplasmic sleeve (Fig. 3b) but in contrast with COL plasmodesmata, it was only occasionally spanned by spokelike elements, and often presented subsections with direct contact between the desmotubule and the PM (Suppl. Fig. 5; Supplementary movies 6-7). Similar to COL cells,

244 Type I plasmodesmata also had a significantly smaller diameter than Type II $(23 \pm 2.6$ $\mathrm{nm}$ versus $37 \pm 7.2 \mathrm{~nm}$, respectively) (Fig. $3 \mathrm{~d}$ ). They also displayed a remarkably constant diameter (PM-PM; inner leaflets) along their entire length, which never varied by more than $5 \mathrm{~nm}$. This contrasted with Type II, whose diameter could range from 25 $\mathrm{nm}$ to more than $40 \mathrm{~nm}$ within a single channel (Fig. 3e). Altogether our data support the view that Type I plasmodesmata present a desmotubule tightly apposed against the PM along the entire length of the pores and that these non-canonical plasmodesmata are not unique to CCI cells.

We next investigated whether Type I and Type II plasmodesmata in cultured cells were in open or closed configurations, or whether these morphotypes corresponded to two populations with distinct internal features. In order to visualise dynamics of 
cells at four, six and thirteen days after sub-culturing. At four days old, when the cells

257 were at the beginning of the linear growth phase, we observed a majority of 258 plasmodesmata with "opaque" appearance (77\% against $23 \%)$, similar to CCI 259 plasmodesmata. With cell ageing, the relative proportion of Type I and Type II was 260 reversed and the majority of the pores had apparent cytoplasmic sleeves $(72 \%$ of Type 261 II at six-day-old) (Fig.3 f-g). This transition happened relatively quickly, between days 262 four and six of cell culture. Consistent with these data, quantitative analyses showed that 263 Type I plasmodesmata were preferentially associated with thin $(101 \pm 48 \mathrm{~nm})$, 264 presumably newly formed cell walls, whereas Type II appeared in thicker (202 $\pm 78 \mathrm{~nm})$, 265 presumably older, cell walls (Fig. 3h).

266 Our data suggest that Type I and Type II plasmodesmata correspond to two 267 distinct morphotypes whose appearance is not only correlated with tissue 268 differentiation but also cell ageing and/or cell wall modification. Type I occur in post269 cytokinesis plasmodesmata and display unconventional structural features 270 characterised by intimate membrane contact between the desmotubule and the PM 271 along the entire length of the channels.

\section{ER-PM contacts are likely to be established during cell plate biogenesis and may} 274 lead to Type I plasmodesmata.

Our data suggest that newly formed plasmodesmata can exhibit very close ER-PM appositions but it is not clear whether such intimate membrane contacts arise during the pore formation or are established post-cytokinetically. We therefore captured plasmodesmata biogenesis events in meristematic epidermal root cells (Fig. 4). As reported in previous studies ${ }^{61,62}$ the earliest traceable event of plasmodesmata formation corresponded to ER membranes perforating the cell plate during the planar fenestrated sheet stage (Fig. 4a-f). At this early stage, the gaps within the fenestrated membrane network were still large $(57.3 \pm 19.7 \mathrm{~nm}, \mathrm{n}=8)$ compared to plasmodesmata

283 diameter and the traversing ER strands were non-constricted with apparent lumen $284(23.5 \pm 4.8 \mathrm{~nm}, \mathrm{n}=8)$. However, regardless of the non-constricted appearance of the ER, intimate ER-PM contacts were already evident at this stage (Fig. 4d-f, red arrows). As the gaps within the fenestrated sheet became narrower, the ER bilayers appressed and tight ER-PM contacts on both sides of the ER were more prominent (Fig. 4g-l). 
Our observations are consistent with an ER-PM attachment occurring in early stages of plasmodesmata biogenesis, before fenestrae closure and ER "entrapment".

Type I plasmodesmata with no visible cytoplasmic sleeve allows non-selective molecule diffusion.

Current models for cell-to-cell trafficking postulate that there is a direct link between ER-PM spacing and plasmodesmata permeability 2,36,37,49. Surprisingly, our data revealed that newly formed plasmodesmata (Type I) are narrow with no apparent cytoplasmic sleeve. We therefore evaluated their permeability.

First, we focused on the CCI/C1 interface and used fluorescence recovery after photobleaching (FRAP) after loading the cells with carboxyfluorescein diacetate (CFDA), a membrane permeant fluorophore that is cleaved by intracellular esterases, yielding a membrane impermeant form of the probe63 (Fig. 5a-c). We bleached a row of cells including the CCI (see Fig. 5a), the QC and adjacent lateral root cap cells to minimize recovery from lateral cells and isolate the CCI-C1 interface. After photobleaching we observed a fast recovery within the CCI suggesting that the probe was able to rapidly diffuse (half time recovery was of 9.7 seconds; $n=10$ ), through the $\mathrm{CCI} / \mathrm{C} 1$ interface, which contain only Type I plasmodesmata (Fig. 5c).

As CFDA has an estimated Stokes radius of only $0.61 \mathrm{~nm}^{64}$, we next investigated the cell-to-cell diffusion of GFP, which has a Stokes radius of $2.82 \mathrm{~nm}^{65}$. To do so, we used Arabidopsis plants expressing GFP under the control of the phloem specific promoter SUC266. In such lines, GFP expression driven by the SUC2 promoter serves as a marker for non-targeted macromolecular movement ${ }^{9,11}$ as it is expressed in the phloem companion cells (Fig. 5d, green) and then diffuses in the neighbouring tissues when plasmodesmal permeability allows it. GFP fluorescence measurements show that GFP was able to diffuse into the root tip including the CCI cells that are exclusively surrounded by Type I plasmodesmata (Fig. 5e,f). Additionally, we measured a gradual 316 decrease of fluorescence from the CCI to the outermost columella tiers (2 fold reduction; 317 Fig. 5f), where only Type II plasmodesmata are found.

318 We could also observe that the transversal walls of root epidermal cells in the 319 meristematic and division zone exclusively harboured Type I plasmodesmata (Fig.5g; $\mathrm{n}$ 320 = 12). To confirm whether or not macromolecules can diffuse through Type I 321 plasmodesmata, we used photo-activable GFP (35S::PA-GFP) and specifically activated 
322 the GFP in one cell and monitored its spread in neighbouring cells. Within few minutes 323 cytoplasmic GFP fluorescence was apparent in neighbouring epidermal cells, indicating 324 symplastic movement across the apico-basal walls.

325 Our results demonstrate that although Type I plasmodesmata have no apparent 326 cytoplasmic sleeve, they can nevertheless allow cell-to-cell movement of 327 micromolecules and macromolecules. 
The structural analysis of plasmodesmata channels is a formidable challenge 330 given their nanoscopic size, location in the cell wall and dynamic nature. Using electron 331 tomography of high pressure-frozen, near-native plasmodesmata, we resolved their 332 ultrastructure at an unprecedented level of 3D detail. Our results revealed unforeseen 333 architectural changes during cellular differentiation and tissue growth, with 334 considerable modification in the internal organisation of these specialised membrane 335 junctions. Based on our observation, we propose a model where archetypal 336 plasmodesmata (Type II), harbouring a cytoplasmic sleeve and spoke-elements, derive 337 from the unconventional Type I plasmodesmata established during cell plate formation. 338 Contrarily to the archetypal model, Type I plasmodesmata present a remarkably close apposition between the desmotubule and the PM to such extent that no visible intermembrane space remains. Type I plasmodesmata we observed resemble, in their ultrastructure and size, pores previously reported in various species such as $N$. 342 tabaccum, $A$. pinnata and B. oleracea, suggesting these may in fact be common 343 structures ${ }^{14,39,67}$. Such proximity between membranes is uncommon amongst MCS 344 structures, where the intermembrane spacing usually varies from 10 to $30 \mathrm{~nm}^{23,25,68}$ and most likely membrane-bridging complexes are required to maintain this minimum gap ${ }^{69}$.

346 Based on our measurements we propose that a $\sim 2-3 \mathrm{~nm}$ wide electron-dense protein 347 meshwork, associated with the desmotubule/PM interfaces, stabilises the contact and perhaps prevents the membranes from repelling each other as such an intimate membrane apposition is thermodynamically unstable ${ }^{69}$. Why are the two membranes in such close contact in Type I plasmodesmata remains to be elucidated. One possibility is that it could favour direct and rapid exchanges of molecules for the establishment of 352 specialised membrane domains during early stages of plasmodesmata formation. We 353 observed Type I plasmodesmata in young walls of Arabidopsis cultured cells, CCI and 354 epidermal root cells, and we propose that these may actually be the predominant 355 morphotype in young/meristematic tissues. As a matter of fact when going back to 356 previous TEM reports on plasmodesmata structure, pores that resemble Type I 357 plasmodesmata have been observed, very often in similar young, meristematic, sink 358 tissues where walls are more likely to be newly divided $14,34,39,40,52,70,71$. Only later in cell 359 development, the gap between the ER and the PM enlarges to form a cytoplasmic sleeve 360 leading to the archetypal Type II plasmodesmata. As seen in the root, this "opening" of 
361 the sleeve can occur quickly, on the opposite cell walls of a single cell. Our model depicts 362 a desmotubule detaching from the PM, first in limited areas and then along the entire 363 length of the channel. In advanced stages of plasmodesmata maturation, the 364 desmotubule is centrally positioned and spokes are numerous and regularly spaced. 365 These correspond to the canonical model of plasmodesmata ultrastructural organisation 366 and have been observed many times in previous TEM studies although not with that 367 level of detail $14,36,39,40,72,73$.

368 A great variety of MCS exist in yeast, animal and plant cells, many of which have 369 been shown to be controlled by tethering proteins or protein complexes $^{74}$ that can 370 appear as electron-dense filament on electron micrographs ${ }^{68}$. These tethering elements 371 physically and functionally connect the two opposing membranes. Tether-like structures 372 are clearly visible in Type II plasmodesmata in the form of spoke-like elements, of about $3739 \mathrm{~nm}$ in length, contacting the desmotubule and the PM. Their appearance coincides with 374 the opening of the cytoplasmic sleeve and the adjustment of the intermembrane gap. 375 This indicates the plasmodesmata tethers/spokes are likely to control ER-to-PM spacing 376 throughout the pore maturation. At this stage it is not clear whether these spokes are 377 already present at early stages of plasmodesmata formation, then unfolded. These 378 plasmodesmata spokes have been observed before by classic TEM and have been 379 thought to be F-actin-associated proteins such as myosin VIII ${ }^{46-54}$. However, our data 380 suggest that membrane tethering at Type II plasmodesmata is not sensitive to destabilization by F-actin polymerization inhibitor drugs. We nevertheless cannot completely rule out that actin in plasmodesmata is unattainable by the drugs. Latrunculin $\mathrm{B}$ and Cytochalasin $\mathrm{D}$ are 0.4 and $0.5 \mathrm{kDa}$ respectively and most certainly pass through plasmodesmata as compounds with similar molecular weights, such as carboxyfluorescein, $\mathrm{do}^{75}$. This suggests that the drugs can indeed physically pass through the pores and reach putative F-actin. However, plasmodesmata may contain very stable actin-associated structures that are not affected by the inhibitors of actin polymerization in our experimental conditions. Nevertheless, our results corroborate the alternative model introduced in 2011 by Tilsner et al. ${ }^{15}$ questioning the presence of 390 F-actin within the plasmodesmal pores due to sterical constraints and conveying a 391 model where spokes are cytoskeleton-independent molecules. In any case, the identity of these plasmodesmata MCS tethers currently remains unanswered. 
Unexpectedly, our data revealed that narrow, newly formed Type I

394 plasmodesmata with no apparent cytoplasmic sleeve nonetheless enable fast small

395 molecule diffusion and even non-selective macromolecule trafficking between cells 396 which appears counterintuitive based on their morphology. These results are however 397 consistent with previous data showing that i) young and meristematic sink tissues with 398 newly divided cell walls harbour plasmodesmata reminiscent of the Type I 399 morphology $14,34,39,40,70,71,76$, and ii) Such young,sink or meristematic tissues often display 400 higher symplastic connectivity than more mature (source tissues), allowing for instance 401 free GFP movement from cell-to-cell, whereas source tissues do not ${ }^{34,35,66,77,78}$. This 402 raises the question of how macromolecules can move through Type I plasmodesmata 403 with such tight cytoplasmic sleeves. Regardless of the answer, our work forces a re404 consideration of how trafficking is achieved in newly formed Type I plasmodesmata.

Altogether, our findings show that ER-PM contacts within plasmodesmata are 406 dynamic and differentially regulated during tissue development and populated by either 407 different tethering molecules and/or different tether conformations at various stages of 408 plasmodesmata maturation. From our data it is clear the PM and desmotubule are 409 always in intimate connection within the pores either through very tight membrane 410 contacts or through spoke-like tethering elements. Similar to other MCS, the function of 411 ER-PM may primarily be the exchange of molecules between the two organelles ${ }^{23,24,30 \text {, }}$ 412 which would then affect plasmodesmata function. In this context, the strikingly different 413 ER-PM connections in Type I and Type II plasmodesmata may have profound 414 implications for intermembrane exchanges and the regulation of plant cell-cell 415 communication. 


\section{Biological material and growth conditions}

429 Six day-old Arabidopsis (Columbia) root tips were grown vertically under greenhouse 430 conditions on solid medium composed of Murashige and Skoog (MS) medium including 431 vitamins $(2.15 \mathrm{~g} / \mathrm{L})$, MES (0.5g/L) and plant-Agar $(7 \mathrm{~g} / \mathrm{L}), \mathrm{pH}$ 5.7. Growth conditions were set at $22^{\circ} \mathrm{C}$ in a greenhouse with a long day $16 \mathrm{~h}$ photoperiod $(100 \mu \mathrm{E} / \mathrm{m} / \mathrm{s})$. Arabidopsis (Landsberg erecta) culture cells were cultivated as described in 22 under constant light $(20 \mu \mathrm{E} / \mathrm{m} / \mathrm{s})$ at $22^{\circ} \mathrm{C}$. Cells were used for experimentation at various ages ranging from four to thirteen-day-old (always mentioned in experiments). For the establishment of a growth curve 5 independent liquid cultures were grown in the same conditions. $2 \mathrm{~mL}$ of culture was sampled every day and the fresh weight was monitored (without the growth medium).

\section{Cryofixation and freeze-substitution}

$441200 \mu \mathrm{m}$ deep and $1.5 \mathrm{~mm}$ wide copper platelets were rapidly filled with either fresh cultured cell clusters or sectioned seedling root tips (approximately $1 \mathrm{~mm}$ in length). These platelets are beforehand coated with 1\% phophastidyl-choline, hexadecene and the bottom is covered with a $50 \mu \mathrm{m}$ thick aclar disk. Additional BSA 20\% was also added in the platelet as a cryoprotectant filler. The prepared platelets containing the samples were then frozen with an EMPACT1 high pressure freezer (Leica, Vienna, Austria). The platelets were then transferred at $-90^{\circ} \mathrm{C}$ into an AFS 2 freeze-substitution machine (Leica, Vienna, Austria) and incubated into a cryosubstitution mix: glutaraldehyde 0.5\%, osmium tetroxide $2 \%$, uranyl acetate $0.1 \%$ and water $1 \%$ in pure acetone. The incubation is only carried out at very low temperature $\left(-90^{\circ} \mathrm{C}\right)$ for $48 \mathrm{~h}$. Then a progressive raise of the temperature of $3^{\circ} \mathrm{C} / \mathrm{h}$ is initiated until $-50^{\circ} \mathrm{C}$ is reached. The cryofixation mix is then carefully and thoroughly removed by 3 consecutive pure acetone washes followed by 3 pure ethanol washes. This very low temperature staining procedure produces a fine membrane staining allowing an improved contrast and resolution, suitable for the observation of nanometric details in electron tomography. To improve embedding, the samples were then carefully removed from the copper platelets and consecutively incubated in HM20 Lowicryl resin (Electron Microscopy Science, EMS) solutions of increasing concentration (dilutions done in pure ethanol): $25 \%$ and $50 \%$ (2 
hours each), 75\% (overnight), 100\% (twice for 2 hours) and a final 4 day incubation in

460 HM20 100\% under UV light. The two first days of incubation were done at $-50^{\circ} \mathrm{C}$ then 461 temperature was quickly risen up to $+20^{\circ} \mathrm{C}$ for the last two days. The use of such an 462 electron lucent resin allowed us to reduce electronic scattering (hence noise) caused by 463 resin-electron interaction, thus improving the $\mathrm{x}, \mathrm{y}, \mathrm{z}$ resolution.

\section{Electron microscopy acquisitions}

Cylindrical moulds (Leica, Vienna, Austria) were used to produce the blocks primarily 467 because they made the production of longitudinal root sections easier. 468 Sections were collected with an Ultracut S (Leica, Vienna, Austria). The sections used 469 ranged from $90 \mathrm{~nm}$ to $180 \mathrm{~nm}$ depending on the volume to acquire. To prevent grid bars from blocking the image, $2 \times 1 \mathrm{~mm}$ oval slot grids filmed with formvar and carbon coated

471 (Electron Microscopy Science, EMS) were used for section collection. Prior to observation, 472 the grids are coated on both sides with $5 \mathrm{~nm}$ gold fiducials (essentially old immunogold 473 secondary antibodies at a dilution ranging from $1 / 20$ to $1 / 100$ or a $1: 1$ mixture of $0.5 \%$ 474 BSA and $5 \mathrm{~nm}$ colloidal gold solution from BBI solution EM-GC5) for the subsequent 475 alignment step.

476 Observations were carried out on a FEI TECNAI Spirit $120 \mathrm{kV}$ electron microscope 477 equipped with a -70 to $+70^{\circ}$ tilting goniometer. A tomography optimised single tilt 478 specimen holder (Fichione instruments, model-2020) was also used to improve the tilting 479 range. The tilt series of longitudinal views of plasmodesmata were acquired at magnifications ranging from $x 30,000$ to $x 56,000$, with images taken each degree. The batch mode special feature of the FEI 3D-explore tomography software allowed us to designate objects of interests, which were then acquired via an automated tilt series data collection process. This technique improved the throughput of our electron tomography workflow allowing us to acquire up to 10 tilt series overnight.

\section{Tomogram reconstruction}

487 The raw $4 \mathrm{k}$ by $4 \mathrm{k}$ pixels tilt series collected need to be aligned before reconstruction.

488 For this manner, two strategies were used : fiducial-less alignment with TomoJ, an 489 Image J plug-in 79,80 and fiducial alignment with eTomo, a graphic interface allowing the 490 use of the IMOD tilt series processing package Etomo 491 (http://bio3d.colorado.edu/imod/). With Etomo, 20 to 30 fiducials in the field of view 
were used to correctly align all images. Good tilt series usually yielded errors of $\approx 1.2$ pixels and below. Aligned stacks were binned two times before reconstruction to make data handling easier. Reconstruction was performed either by using the weighed backprojection or the SIRT algorithm (15 to 20 iterations with default parameters) of Etomo or the OSART iterative algorithm of TomoJ (100 iterations, 0.01 as relaxation coefficient, update every 5 images). Optional 2D filtering of the aligned stack performed prior to reconstruction with the default parameters of Etomo 0.35 cut-off and 0.05 sigma roleoff) efficiently filtered the highest frequencies, rendering less noisy tomograms when needed. This extra filtering step allowed the SIRT algorithm of Etomo to yield more contrasted tomograms.

Combination of tomograms in the case of dual-tilt axis tomography was performed using Etomo with default parameters. Both single tilt tomograms were generated as described above. The cell wall region was eventually excluded from the processing as it increased the correlation error scores because of its lack of electron-dense features necessary for the patch correlation step.

\section{Image segmentation and tomogram analysis}

Manual segmentation of tomograms was performed with $3 \mathrm{dmod}$ and allowed us to visualise and appreciate the organisation of the sub-elements in a 3D space. Additionally, an efficient way of segmenting structures was to accurately outline their main contours throughout the volume using the interpolation tool (drawing tools IMOD package developed by Andrew Noske) and then generate an isosurface of the structure.

Systematic measurements were taken on all relevant raw tomograms (without filters applied to minimize measurement errors), comprising width of the desmotubule, plasmodesmata channel, cell wall thickness at the pores, length of the spokes if visible etc. Pore width, cytoplasmic sleeve space and spoke length were measured relative to the inner leaflets of the PM (facing the symplasm). The desmotubule was always measured in sections where it was clearly distinguishable and at its largest. Inter-spoke spacing, shape of spokes were also accounted for in relevant tomograms where spokes were clearly distinguishable. 
525 TEM plasmodesmata Type I and II screening method

526 For Fig. 3 panels $\mathrm{h}$ and g, fractions of Type I and type II plasmodesmata were assessed 527 by TEM in order to raise statistically significant numbers. This was possible because 528 Type I and Type II plasmodesmata could be discriminated by classic TEM (Suppl. Fig. 8). 529 To avoid potential sampling artefacts, a stereology based approach inspired from the 530 dissector method 81,82 was used. Two to three biological replicates were used for 531 plasmodesmata screening in culture cells for each time point.

532 Counting was done on $90 \mathrm{~nm}$ serial sections. Preliminary measures showed that only $53320 \%$ of the plasmodesmata spotted on a reference section $n$ could be followed on the 534 contiguous lookup section $n+1$, meaning that $80 \%$ of the plasmodesmata spotted on a 535 single section were "resolved". The counting was therefore performed on sections $n$ and $536 n+2$ to avoid any kind of double counting therefore giving more weight to larger pores. 537 Plasmodesmata were considered resolved when the PM bilayers were clearly 538 distinguishable.

\section{Drug treatment}

541 10mM/DMSO stock solutions of actin destabilizing agents latrunculin B (Calbiochem, $5421 \mathrm{mg}$ ) and cytochalasin D (Sigma-aldrich, 1mg) were used at a working concentration of $54350 \mu \mathrm{m}$ (dilution in liquid MS medium) for $1 \mathrm{~h} 30 \mathrm{~min}$. Controls contained diluted DMSO 544 only.

\section{Permeability measurements}

547 FRAP/CFDA: Plasmodesmal permeability assessments were made using FRAP on six 548 day-old Arabidopsis root tips co-stained with CFDA (50 $\mu \mathrm{g} / \mathrm{mL})$ and Propidium iodide. 549 Roots were incubated in an aqueous CFDA solution for 5 minutes, then successively 550 washed out in 3 water baths and mounted with propidium iodide in water for imaging. 551 Acquisitions were made on a Zeiss LSM 880 equipped with an Argon laser (excitation $488 \mathrm{~nm}$ ) and a 40x apochromate 1.30 oil-objective. In order to optimize the frame rate,

553 cropping of the scanned area was done very consciously by limiting the height of the 554 scanned area as much as possible and enlarging it in order to decrease the scanning time 555 ( $\approx 90 \mathrm{~ms}$ at max scanning speed) while having access to the background, and 556 neighbouring cells relative to the photobleached region. To assess the permeability 557 specifically at the CCI-C1 interface, the photobleaching region consisted in a rectangle 
558 encompassing CCI, QC cells and the surrounding initial cells, while recovery was 559 monitored in the CCI only. This allowed us to isolate and measure fluorescence recovery 560 in a unidirectional fashion. The FRAP routine consisted in 10 images pre-bleach at 20\% 561 laser power and max scanning speed (reaching $\approx 90 \mathrm{~ms}$ per image), ten iterations of 562 photobleaching with $100 \%$ of $488 \mathrm{~nm}$ laser where pixel dwell time was increased to a 563 value of $\approx 1 \mu \mathrm{s} /$ pixel. $\approx 400$ post-bleach images were then acquired in order to reach the 564 stationary phase of the fluorescence recovery with the same parameters than the pre565 bleach images. The recovery profiles were accounted for noise and then double 566 normalized and set to full scale mode (pre-bleach is set to 1 and first image post-bleach 567 is set to 0) as described by Kote Miura in his online FRAP-teaching module (EAMNET568 FRAP module, https://www.embl.de). Plotting and curve fitting was done using 569 GraphPad Prism (GraphPad Software, Inc).

570 pSUC2::GFP root: Regions of interest of same dimensions were used to measure the 571 fluorescence intensity in multiple regions of six day-old pSUC2::GFP roots (vasculature, 572 CCI and C1-CO columella layers) using confocal microscopy. The fluorescence intensities 573 were then normalized relative to the intensity measured in the root vasculature. 574 Background noise was substracted using wild type roots. Co-staining with propidium iodide allowed an easy visualization of the cellular organization of the root tip, thus

576 allowing precise fluorescence intensity measurements in the different cells of interest.

577 Photoactivation in the root epidermis using 35S::PA:GFP lines : Six days old 35S::PA:GFP

578 arabidopsis roots were imaged using a Zeiss LSM880 confocal laser scanning microscope with $63 \mathrm{x}$ oil lens. Propidium iodide was excited at $488 \mathrm{~nm}$ with $10 \%$ of laser power and fluorescence collected at 590-650 nm. PA-GFP was activated at $405 \mathrm{~nm}$ with $3 \%$ of laser power and fluorescence emission collected at 505-550 nm with $10 \% 488 \mathrm{~nm}$

582 laser power. Photoactivation was done in single epidermal cell of the meristematic zone, 583 where type I plasmodesmata were observed on the transversal walls. Both GFP and 584 propidium iodide fluorescence were acquired every five minutes during 25 minutes. 585 Quantification was done using Zenblue 2012 (Zeiss) in the activated and neighbouring 586 cells (proximal and proximal+1) to assess GFP diffusion through apico-basal walls. 587 Fluorescence intensity in the different cells was expressed as a percentage of total 588 fluorescence (activated, proximal, proximal+1). 
591 Data availability

592 The data that support the findings of this study are available from the corresponding 593 author upon request 


\section{BIBLIOGRAPHY}

595 1. Stahl, Y. \& Faulkner, C. Receptor complex mediated regulation of symplastic traffic. Trends Plant Sci. 21, 450-459 (2016).

2. Otero, S., Helariutta, Y. \& Benitez-alfonso, Y. Symplastic communication in organ formation and tissue patterning. Curr. Opin. Plant Biol. 29, 21-28 (2016).

599

3. Benkovics, A. H. \& Timmermans, M. C. P. Developmental patterning by gradients of 600 mobile small RNAs. Curr. Opin. Genet. Dev. 27, 83-91 (2014).

601

4. Vaddepalli, P. et al. The C2-domain protein QUIRKY and the receptor-like kinase

602

603

604 STRUBBELIG localize to plasmodesmata and mediate tissue morphogenesis in Arabidopsis thaliana. Dev 141, 4139-4148 (2014).

605

606

607

608

609

5. Daum, G., Medzihradszky, A., Suzaki, T. \& Lohmann, J. U. A mechanistic framework for noncell autonomous stem cell induction in Arabidopsis. Proc. Natl. Acad. Sci. U. S. A. 111, 14619-24 (2014).

6. Stahl, Y. et al. Moderation of arabidopsis root stemness by CLAVATA1 and ARABIDOPSIS CRINKLY4 receptor kinase complexes. Curr. Biol. 23, 362-371 (2013).

7. Benitez-alfonso, Y. et al. Symplastic intercellular connectivity regulates lateral root patterning. Dev. Cell 26, 136-47 (2013).

8. Faulkner, C. et al. LYM2-dependent chitin perception limits molecular flux via plasmodesmata. Proc. Natl. Acad. Sci. U. S. A. 110, 9166-70 (2013).

9. Wu, S. et al. Symplastic signaling instructs cell division, cell expansion, and cell polarity in the ground tissue of Arabidopsis thaliana roots. Proc. Natl. Acad. Sci. (2016).

10. Lee, J.-Y. \& Lu, H. Plasmodesmata: the battleground against intruders. Trends Plant Sci. 16, 201-10 (2011).

11. Vatén, A. et al. Callose biosynthesis regulates symplastic trafficking during root development. Dev. Cell 21, 1144-55 (2011).

12. Tilsner, J. et al. Replication and trafficking of a plant virus are coupled at the entrances of plasmodesmata. J. Cell Biol. 201, 981-95 (2013).

13. Benitez-Alfonso, Y., Faulkner, C., Ritzenthaler, C. \& Maule, A. J. Plasmodesmata: gateways to local and systemic virus infection. Mol. Plant. Microbe. Interact. 23, 1403-12 (2010).

626 14. Ding, B., Turgeon, R. \& Parthasarathy, M. V. Substructure of freeze-substituted 
plasmodesmata. Protoplasma 169, 28-41 (1992).

15. Tilsner, J., Amari, K. \& Torrance, L. Plasmodesmata viewed as specialised membrane adhesion sites. Protoplasma 248, 39-60 (2011).

16. Knox, K. et al. Putting the squeeze on plasmodesmata: A role for RETICULONs in primary plasmodesmata formation. Plant Physiol. 168, 1563-72 (2015).

17. Salmon, M. S. \& Bayer, E. M. F. Dissecting plasmodesmata molecular composition by mass spectrometry-based proteomics. Front. Plant Sci. 3, (2012).

18. Simpson, C., Thomas, C., Findlay, K., Bayer, E. \& Maule, A. J. An Arabidopsis GPI-

19. Thomas, C. L., Bayer, E. M., Ritzenthaler, C., Fernandez-Calvino, L. \& Maule, A. J. anchor plasmodesmal neck protein with callose binding activity and potential to regulate cell-to-cell trafficking. Plant Cell 21, 581-94 (2009).

20. Fernandez-Calvino, L. et al. Arabidopsis plasmodesmal proteome. PLoS One 6, (2011).

25. Gallo, A., Vannier, C. \& Galli, T. Endoplasmic Reticulum-Plasma Membrane Associations:Structures and Functions. Annu. Rev. Cell Dev. Biol. 32, 279-301 (2016).

26. Filseck, J. M. Von et al. Phosphatidylserine transport by ORP/Osh proteins is driven by phosphatidylinositol 4-phosphate. Science 349, 432-36 (2015).

27. Chang, C. L. et al. Feedback regulation of receptor-induced ca2+ signaling junctions. Cell Rep. 5, 813-825 (2013). 
660

661

662

663

664

665

666

667

668

669

670

671

672

673

674

675

676

677

678

679

680

681

682

683

684

685

686

687

688

689

690

691

692

28. Giordano, F. et al. PI(4,5)P2-Dependent and Ca2+-Regulated ER-PM interactions mediated by the extended synaptotagmins. Cell 153, 1494-1509 (2013).

29. Chu, B.-B. et al. Cholesterol Transport through Lysosome-Peroxisome Membrane Contacts. Cell 161, 291-306 (2015).

30. Tilsner, J., Nicolas, W., Rosado, A. \& Bayer, E. M. Staying tight: plasmodesmata membrane contact sites and the control of cell-to-cell connectivity. Annu. Rev. Plant Biol. 67, 23.1-23.28 (2016).

31. Gisel, A., Barella, S., Hempel, F. D. \& Zambryski, P. C. Temporal and spatial regulation of symplastic trafficking during development in Arabidopsis thaliana apices. Dev 126, 1879-1889 (1999).

32. Sivaguru, M. et al. Aluminum-induced 1,3-beta-D-glucan inhibits cell-to-cell trafficking of molecules through plasmodesmata. A new mechanism of aluminum toxicity in plants. Plant Physiol. 124, 991-1006 (2000).

33. Li, W. et al. Callose deposition at plasmodesmata is a critical factor in restricting the cell-to-cell movement of Soybean mosaic virus. Plant Cell Rep. 31, 905-16 (2012).

34. Oparka, K. J. et al. Simple, but not branched, plasmodesmata allow the nonspecific trafficking of proteins in developing Tobacco leaves. Cell 97, 743-54 (1999).

35. Kim, I. \& Zambryski, P. C. Cell-to-cell communication via plasmodesmata during Arabidopsis embryogenesis. Curr. Opin. Plant Biol. 8, 593-9 (2005).

36. Schulz, A. Plasmodesmata widening accompanies the short-term increase in symplasmic phloem unloading in pea root tips under osmotic stress. Protoplasma 188, 22-37 (1995).

37. Brunkard, J. O., Runkel, A. M. \& Zambryski, P. C. The cytosol must flow: intercellular transport through plasmodesmata. Curr. Opin. Cell Biol. 35, 13-20 (2015).

38. Beck, M. et al. Nuclear pore complex structure and dynamics revealed by cryoelectron tomography. Science 306, 1387-90 (2004).

39. Overall, R. L., Wolfe, J. \& Gunning, B. E. S. Intercellular communication in Azolla roots: I. Ultrastructure of plasmodesmata. Protoplasma 111, 134-150 (1982).

40. Tilney, L. G., Cooke, T. J., Connelly, P. S. \& Tilney, M. S. The structure of plasmodesmata as revealed by plasmolysis, detergent extraction, and protease digestion. J. Cell Biol. 112, 739-47 (1991). 
41. Moore, R., Fondren, W. M., Koon, E. C. \& Wang, C. L. The influence of gravity on the formation of amyloplasts in columella cells of Zea mays L. Plant Physiol. 82, 867868 (1986).

42. Bennett, T. et al. SOMBRERO, BEARSKIN1, and BEARSKIN2 regulate root cap maturation in Arabidopsis. Plant Cell 22, 640-654 (2010).

43. Staehelin, L. a, Zheng, H. Q., Yoder, T. L., Smith, J. D. \& Todd, P. Columella cells revisited: novel structures, novel properties, and a novel gravisensing model. Gravit. Space Biol. Bull. 13, 95-100 (2000).

44. Dolan, L. et al. Cellular-Organization of the Arabidopsis-Thaliana Root. Dev 119, 71-84 (1993).

45. Hamamoto, L., Hawes, M. C. \& Rost, T. L. The production and release of living root cap border cells is a function of root apical meristem type in dicotyledonous angiosperm plants. Ann. Bot. 97, 917-923 (2006).

46. Overall, R. L. \& Blackman, L. M. A model of the macromolecular structure of plasmodesmata. Trends Plant Sci. 1, 307-311 (1996).

47. Oparka, K. J. Getting the message across: How do plant cells exchange macromolecular complexes? Trends Plant Sci. 9, 33-41 (2004).

48. White, R. \& Barton, D. The cytoskeleton in plasmodesmata: a role in intercellular transport? J. Exp. Bot. 62, 5249-5266 (2011).

49. Radford, J. E. \& White, R. G. Inhibitors of myosin, but not actin, alter transport through Tradescantia plasmodesmata. Protoplasma 248, 205-16 (2011).

50. Mongrand, S., Stanislas, T., Bayer, E. M. F., Lherminier, J. \& Simon-Plas, F. Membrane rafts in plant cells. Trends Plant Sci. 15, 656-63 (2010).

51. Sevilem, I., Yadav, S. R. \& Helariutta, Y. Plasmodesmata : Channels for intercellular signaling during plant growth and development. 1217, 3-24 (2015).

52. White, R. G. et al. Actin associated with plasmodesmata. Protoplasma 180, 169184 (1994).

53. Radford, J. E. \& White, R. G. Localization of a myosin-like protein to plasmodesmata. Plant J. 14, 743-50 (1998).

54. Faulkner, C. R., Blackman, L. M., Collings, D. a, Cordwell, S. J. \& Overall, R. L. Antitropomyosin antibodies co-localise with actin microfilaments and label plasmodesmata. Eur. J. Cell Biol. 88, 357-69 (2009).

55. Ding, B., Kwon, M. \& Warnberg, L. Evidence that actin filaments are involved in 
controlling the permeability of plasmodesmata in Tobacco mesophyll. Plant J. 10, 157-164 (1996).

56. Sheahan, M. B., Staiger, C. J., Rose, R. J. \& McCurdy, D. W. A green fluorescent protein fusion to actin-binding domain 2 of Arabidopsis fimbrin highlights new features of a dynamic actin cytoskeleton in live plant cells. Plant Physiol. 136, 3968-3978 (2004).

57. Boutté, Y. et al. Endocytosis restricts Arabidopsis KNOLLE syntaxin to the cell division plane during late cytokinesis. EMBO J. 29, 546-58 (2010).

58. Marais, C. et al. The Qb-SNARE Memb11 interacts specifically with Arf1 in the Golgi apparatus of Arabidopsis thaliana. J. Exp. Bot. 66, 6665-6678 (2015).

59. Bradley, M. O. Microfilaments and cytoplasmic streaming: Inhibition of streaming with cytochalasin. J. Cell Sci. 12, 327-343 (1973).

60. Bayer, E., Thomas, C. L. \& Maule, A. J. Plasmodesmata in Arabidopsis thaliana suspension cells. Protoplasma 223, 93-102 (2004).

61. Hawes, C. R., Juniper, B. E. \& Horne, J. C. Low and High voltage electron microscopy of mitosis and cytokinesis in maize roots. Planta 152, 397-407 (1981).

62. Hepler, P. K. Endoplasmic reticulum in the formation of the cell plate and plasmodesmata. Protoplasma 111, 121-133 (1982).

63. Rutschow, H. L., Baskin, T. I. \& Kramer, E. M. Regulation of solute flux through plasmodesmata in the root meristem. Plant Physiol. 155, 1817-26 (2011).

64. Wang, N. \& Fisher, D. B. The Use of Fluorescent Tracers to Characterize the Post-

65. Terry, B. R., Matthews, E. K. \& Haseloff, J. Molecular characterization of recombinant green fluorescent protein by fluorescence correlation microscopy. Biochem. Biophys. Res. Commun. 217, 21-27 (1995).

67. Blackman, L. M., Harper, J. D. I. \& Overall, R. L. Localization of a centrin-like protein to higher plant plasmodesmata. Eur. J. Cell Biol. 78, 297-304 (1999). 
membrane contact sites. Proc. Natl. Acad. Sci. 112, (2015).

760

761

762

763

764

765

766

767

768

769

770

771

772

773

774

775

776

777

778

779

780

781

782

783

784

785

786

787

788

789

790

791

69. Li, F. et al. Energetics and dynamics of SNAREpin folding across lipid bilayers. Nat. Struct. Mol. Biol. 14, 890-896 (2007).

70. Olesen, P. The neck constriction in plasmodesmata. Planta 358, 349-358 (1979).

71. Burch-Smith, T. M. \& Zambryski, P. C. Loss of increased size exclusion limit (ise)1 or ise 2 increases the formation of secondary plasmodesmata. Curr. Biol. 20, 989993 (2010).

72. Bell, K. \& Oparka, K. Imaging plasmodesmata. Protoplasma 248, 9-25 (2011).

73. Robinson-Beers, K. \& Evert, R. F. Ultrastructure of and plasmodesmatal frequency in mature leaves of sugarcane. Planta 184, 291-306 (1991).

74. Eisenberg-Bord, M., Shai, N., Schuldiner, M. \& Bohnert, M. A Tether Is a Tether is a Tether : Tethering at Membrane Contact Sites. Dev. Cell 39, 395-409 (2016).

75. Duckett, C. M., Oparka, K. J., Prior, D. a M., Dolan, L. \& Roberts, K. Dye-coupling in the root epidermis of Arabidopsis is progressively reduced during development. Dev 120, 3247-3255 (1994).

76. Badelt, K., White, R. G., Overall, R. L. \& Vesk, M. Ultrastructural Specializations of the Cell Wall Sleeve Around Plasmodesmata. Am. J. 81, 1422-1427 (2016).

77. Kim, I., Kobayashi, K., Cho, E. \& Zambryski, P. C. Subdomains for transport via plasmodesmata corresponding to the apical-basal axis are established during Arabidopsis embryogenesis. Proc. Natl. Acad. Sci. 102, 11945-11950 (2005).

78. Kim, I., Cho, E., Crawford, K., Hempel, F. D. \& Zambryski, P. C. Cell-to-cell movement of GFP during embryogenesis and early seedling development in Arabidopsis. Proc. Natl. Acad. Sci. U. S. A. 102, 2227-2231 (2005).

79. Messaoudi, C., Boudier, T., Sanchez Sorzano, C. O. \& Marco, S. TomoJ: tomography software for three-dimensional reconstruction in transmission electron microscopy. BMC Bioinformatics 8, 288 (2007).

80. Sorzano, C. O. S. et al. Marker-free image registration of electron tomography tiltseries. BMC Bioinformatics 10, 124 (2009).

81. Lucocq, J. Unbiased 3-D quantitation of ultrastructure in cell biology. Trends Cell Biol. 3, 354-358 (1993).

82. Sterio, D. C. The unbiased estimation of number and sizes of arbitrary particles using the disector. J. Microsc. 134, 127-136 (1984). 
Figure 1. Plasmodesmata ER-PM contact site morphology evolves during tissue development in root tips.

(a) Schematic representation of Arabidopsis root tip cellular organisation, in which the colours depict cell lineage. Plasmodesmal ultrastructure was studied in the Columella tiers in light red (yellow boxes), from the C1 to C4 layer and in the COL cell initials in red (CCI, yellow asterisks). (b-d) Tomographic slices of representative plasmodesmata from the CCI (b), C1-C2 (c) and C3-C4 (d) interfaces, respectively 0.56, 0.56 and $0.49 \mathrm{~nm}$ thick, and their associated 3D segmentation highlighting the changes in ER-PM architecture within the pores during COL cell differentiation. In CCI, appressed ER is seen entering the plasmodesmal pores (black arrowheads) and fills the entire canal (b). With tissue differentiation the cytoplasmic sleeve becomes gradually visible as the gap between the desmotubule and PM expands and become populated by spoke-like tethers (d, yellow arrows). In the C1/C2 transition zone there is no clearly identifiable spokeelements in the cytoplasmic sleeve, only amorphous material (c, red). (e) Difference in cell wall thickness in CCI, C1-C2 and C3-C4 cells (Dunn's multiple comparison test **** $\mathrm{P}<0.0001$ ). $\mathrm{n}=94$ for CCI, $\mathrm{n}=27$ for C1-C2 and $\mathrm{n}=79$ for C3-C4. (f) Average diameter of plasmodesmata (PM-PM, inner leaflets) and desmotubules in CCI, C1-C2 and C3-C4 cells (Dunn's multiple comparison test, ${ }^{*} \mathrm{P}<0.05,{ }^{* * *} \mathrm{P}<0.001$ ). $\mathrm{n}=20, \mathrm{n}=15$ and $\mathrm{n}=15$ plasmodesmata tomograms for CCI, C1-C2 and C3-C4, respectively. CCI: columella cell initials; CS: cytoplasmic sleeve; Dt: desmotubule; ER: endoplasmic reticulum; QC: quiescent centre; PM: plasma membrane.

Figure 2. The spoke-like tethering elements of Type II plasmodesmata correlate with ER-PM spacing and are not sensitive to F-actin polymerization inhibitor drugs

819 (a-c) $1.24 \mathrm{~nm}$ thick tomographic slices depicting the typical and regular arrangement of 820 the spoke-like tethering elements (yellow arrows) in Type II plasmodesmata, in the root 821 columella (C2-C4). (b) Close-up view of the plasmodesma squared in (a). (c) Manual segmentation of (b). (d) Density representation of the spoke length, measured in COL cells and cultured Arabidopsis cells (non-treated, Latrunculin B, LaB and Cytochalasin D,

824 CytD treated for roots and non treated for cultured cells). In either condition, there is a 
peak density for spokes of about $9 \mathrm{~nm}$ in length. (e) Three close-up views of V/Y shaped spokes (red arrowheads) oriented towards the PM (top; middle) and the desmotubule 827 (bottom). Scale bars $=5 \mathrm{~nm}$. (f) Plot representation of the cytoplasmic sleeve gaps 828 (desmotubule - PM distance) measured in subsections of transitioning or Type II pores with or without spokes. Both in roots and cultured cells the presence of spokes stabilizes the width of the intermembrane gap and keeps it at an average of $9.6 \pm 2.44 \mathrm{~nm}$ whereas without the spokes this gap is $18.38 \pm 7.26 \mathrm{~nm}$ (Mann-Whitney two tailed test, **** $\mathrm{P}<0.0001) \mathrm{n}=31$ and $\mathrm{n}=65$ measurements in cell and root plasmodesmata respectively. (g-l) Type II plasmodesmata in COL cells (C2-C4) are not altered by F-actinpolymerization inhibitor drugs. (g-i) Maximum projections of confocal stacks taken in columellas of Arabidopsis marker lines fimbrin actin binding domain 35S::GFP-fABD2GFP. In control conditions (g), cells show a dense, reticulated actin network, while after $1 \mathrm{~h} 30$ hour treatment with $50 \mu \mathrm{M}$ Cytochalasin D (CytD, h) or Latrunculin B (LaB, i), the F-actin network is heavily altered. (j-k) $0.56 \mathrm{~nm}$ thick tomographic slices of plasmodesmata acquired in the columella of CytD (j) and LaB (k)-treated roots, showing that the spokes are still present. (1) The diameter (PM-PM; innerleaflets) of the pores and the desmotubule width remain unchanged both treatment (Dunn's multiple comparison test, $\left.{ }^{* * * *} \mathrm{P}<0.0001,{ }^{* * *} \mathrm{P}<0.001\right) \mathrm{n}=41$ plasmodesmata tomograms for non-treated (NT) condition, $\mathrm{n}=13$ for $\mathrm{LaB}$ and $\mathrm{n}=16$ for CytD treated condition. Dt: desmotubule ; PM : plasma membrane.

\section{Figure 3. Very tight ER-PM contact in post-cytokinesis plasmodesmata}

847 (a-b) Consecutive tomographic slices, of respectively $1.24 \mathrm{~nm}$ and $0.49 \mathrm{~nm}$ in thickness, 848 of a Type I plasmodesma (a) and Type II plasmodesma (b, spokes are indicated by yellow dashes) in Arabidopsis cultured cells. (c) Type I plasmodesmata are traversed by the ER which becomes appressed just before entering the pores (yellow arrows). Three $0.56 \mathrm{~nm}$ thick tomographic slices and the corresponding 3D segmentation. (d) 852 Plasmodesmata diameter (PM-PM; inner leaflets) of Type I (23 $\pm 2.6 \mathrm{~nm})$ and Type II (37 $853 \pm 7.2 \mathrm{~nm})$, and the desmotubule measured in Type II plasmodesmata $(17 \pm 2.4 \mathrm{~nm}) . \mathrm{n}=$ 85417 and 22 tomograms for Type I and Type II plasmodesmata, respectively, and $\mathrm{n}=22$ for desmotubule measurements ( ${ }^{* * * *} \mathrm{P}<0.0001$ by Mann-Whitney test). (e) plasmodesmata

856 width at different points along the pores in Type I and II. Measurements (PM-PM; inner 857 leaflets) were taken at the extremities and largest part of the channels. Type I 
858 plasmodesmata have a remarkably constant diameter compared to Type II. $\mathrm{n}=17$ and $\mathrm{n}$

$859=22$ tomograms for Type I and Type II plasmodesmata, respectively. (f) Growth curve of 860 Arabidopsis liquid cultured cells. Black arrows indicate the cell culture ages used for this 861 study (four-, six- and thirteen-day-old). (g) Quantification of the relative proportion of 862 Type I and II plasmodesmata in Arabidopsis cultured cells at four, six and thirteen days 863 ( $\mathrm{n}=111,89$ and 22 screened plasmodesmata for four, six and thirteen days old cells). 864 (h) Average cell wall thickness in relation to plasmodesmata Type. In four-day-old 865 cultured cells, Type I plasmodesmata are abundant in thin young cell walls whereas 866 Type II plasmodesmata are preferentially associated with thicker, older walls. $\mathrm{n}=69$ 867 and 28 for Type I and II plamodesmata, respectively (**** $\mathrm{P}<0.0001$ by Mann-Whitney 868 test). For (g) and (f), plasmodesmata screening was done on $90 \mathrm{~nm}$ thick sections by 869 TEM (see M\&M for details). CS: cytoplasmic sleeve; Do: days of culture; Dt: desmotubule; 870 ER: endoplasmic reticulum; PM: plasma membrane.

872 Figure 4. Very tight ER-PM contacts are established during cell plate formation.

873 (a-f) $0.46 \mathrm{~nm}$ thick tomographic slices, and the associated segmentation show non874 appressed ER strands trapped by the fenestrated cell plate, establishing very tight 875 contacts (d-f, red arrows and dashed line) at very early stages of plasmodesmata 876 initiation. (g-l) $0.56 \mathrm{~nm}$ thick $(\mathrm{g}, \mathrm{h})$ and $0.36 \mathrm{~nm}$ thick $(\mathbf{j}, \mathbf{k})$ tomographic slices depicting 877 the establishment of very tight ER-PM contacts occurring on one end of the forming 878 plasmodesma ( $\mathrm{g}$-i, red arrows and dashed line) and along its entire length (j-l, red 879 arrows and dashed line). CP: cell plate; ER: endoplasmic reticulum; PM: plasma 880 membrane.

\section{Figure 5. Molecular trafficking through Type I plasmodesmata}

883 (a-c) Plasmodesmata permeability at the CCI/C1 interface monitored by FRAP and CDFA 884 (a) Col. 0 root tip co-stained with CFDA and Propidium Iodide. Orange and blue (CCI) 885 boxes indicate regions that were photobleached and where fluorescence intensity was 886 monitored, respectively. (b) Representative kymograph of CCI region (blue box in $a$ ). 887 Fire LUT was applied to enhance visualization of the photobleaching and recovery. (c) 888 Mean recovery curve with error bars indicating standard deviation (3 independent 889 experiments; 10 successful FRAPs in 10 individual roots), showing rapid recovery of 
890 CFDA within the CCI. The one-phase exponential association curve fit $\left(\mathrm{R}^{2}=0.86\right)$ 891 calculated a half-time recovery of 9.7 seconds, a K constant of $0.07 \mathrm{~s}^{-1}$ and a Ymax of 0.86 892 (d-f) Non-targeted diffusion of free GFP in the COL cells using pSUC2::GFP lines. (d) 893 Cartoon of Arabidopsis pSUC2::GFP root. Green cells represent the companion cells 894 where the GFP is expressed. The presence of GFP in other parts of the root is due to 895 diffusion through plasmodesmata. Red and blue colours show cell interfaces harbouring 896 Type I or Type II plasmodesmata, respectively. (e) Confocal slices through Col 0 (left panels) and pSUC2::GFP (right panels) root tips exhibiting GFP signal (green) in the columella. Close up view (yellow boxed regions in upper panels) in the meristematic region show the CCI contours in the propidium iodide channel (white stars in the two 900 bottom panels) reveal the CCI cells, right below the periblem layer containing the 901 quiescent centre. GFP signal is visible in the meristematic area of pSUC2::GFP lines, in contrast to the absence of fluorescence in the Col.0 root tip. (f) GFP fluorescent quantification in the pSUC2::GFP lines in CCI, and C1-C4 COL layers (background was subtracted against Col-0 roots). Intensities are normalized within a given root relative to the intensity in the vascular system (set to 1$)$. ( $n=15$ pSUC2::GFP roots and $n=10$ Col. 0 roots in 3 independent experiments). Wilcoxon test was used to compare each cell type to the CCI cells. ${ }^{*} \mathrm{P}<0.05,{ }^{* *} \mathrm{P}<0.01,{ }^{* * *} \mathrm{P}<0.001$.

908 (g-i) Photoactivated (PA)-GFP diffusion through Type I plasmodesmata. (g) Two 0.56 $909 \mathrm{~nm}$ thick tomographic slices of Type I plasmodesmata in transversal walls of epidermal cells in the root meristematic zone. (h) Confocal slices showing PA-GFP signal in 911 photoactivated cell ( $\mathrm{t} 0$; white asterisk) and reaching the neighbouring cells after t25 912 minutes. Right panel represents a color-coded cartoon. (i) Fluorescence was quantified 913 in the photo-activated (blue) and the adjacent ( $n$ proximal, in red and $n+1$ in green) cells.

914 PA-GFP fluorescence in activated cells consistently showed a decrease of intensity over 915 time whereas neighbouring cells $(n, n+1)$ showed a concomitant increase in fluorescence. 916 ( $\mathrm{n}=15$ roots; 5 independent experiments). Two-tailed Wilcoxon test was used to 917 compare the fluorescence intensity in a given cell over time. The subsequent times 918 points after photoactivation were always tested with t0 as the reference, for a given cell. $919 * * \mathrm{P}<0.01$. CCI: columella cell initial; CFDA: carboxyfluorescein diacetate; COL: columella; FRAP: Fluorescence recovery after photobleaching; LUT: look up table. 
923 Supplementary Fig 1.

924 (a, b) $0.56 \mathrm{~nm}$ thick tomographic slices of plasmodesmata found in the longitudinal cell

925 walls of the C2 cell tier showing transition in their architecture between Type I and Type

926 I with spoke-less central cavities. (c) $0.56 \mathrm{~nm}$ thick tomographic slices of a transitioning

927 plasmodesmata at the C2-C3 layers interface. Spokes are starting to appear (white

928 arrowheads) between PM and desmotubule and the cytoplasmic gap is consequently

929 tighter than when spokes are absent. (d) Schematic representation depicting how the

930 measurements were done for figure panel 3f. Measurements were always taken where

931 the width of the gap was at its maximum. Blue double-headed arrow shows a typical

932 measurement done in a spoke-less cavity contributing to the data plotted in panel 3f.

933 Red double headed arrow shows same measurement executed in a cavity spanned by

934 spoke-like tethers. Brown : cell wall ;Green : ER and the derived desmotubule ;Orange :

935 spoke-like tethers.

936

\section{Supplementary movie 2.}

938 Timelapse of Mb12:YFP lines (a-c) and Ng1:GFP lines (d-f) taken at $\mathrm{t}=0,30 \mathrm{~min}$ and $1 \mathrm{~h}$ 939 of treatment at 1 image every 5 seconds. Before treatment (a,d) golgi vesicles are 940 numerous small and rapidely moving within the cells. After LaB (b, e) and CytD (c, f) 941 treatment the vesicles were less mobile and tended to aggregate. The decrease in

942 specific signal over time is due to photobleaching. All images were taken at same 943 magnification under same conditions.

944

945 Supplementary Fig 3.

946 Cultured cells consist of small cell clusters and mostly contain primary plasmodesmata 947 on contact walls that are formed during cytokinesis 60,62 .

948 (a) Overview of Arabidopsis cell clusters in transmitted light. (b) $90 \mathrm{~nm}$ ultrathin section 949 containing cells on a copper EM hexagonal grid. (c) TEM micrograph of a single stranded 950 plasmodesma (white arrow) inserted in a division wall.

952 Supplementary movie 4.

953 A plasmodesma with Type I structure in Arabidopsis cultured cells.

954 The movie displays $0.68 \mathrm{~nm}$ thick tomographic slices and a 3D rendering of the data 955 shown in Fig. 3c. ER/desmotubule in pale blue and PM in orange. Scale bar $=50 \mathrm{~nm}$ 


\section{Supplementary Fig 5.}

958 (a-d) Tomograms of two Type II plasmodesmata from Arabidopsis cultured cells 959 illustrating the fluctuation in the desmotubule to PM gap along the channels and points 960 of very close ER-PM appositions (black arrowheads). The presence of spoke-elements 961 are indicated by yellow arrows. Tomographic slices of $0.56 \mathrm{~nm}$ (a) and $0.46 \mathrm{~nm}$ (c) in 962 thickness, and their corresponding segmentation (b and d). CS: cytoplasmic sleeve; Dt: 963 desmotubule; ER: endoplasmic reticulum; PM: plasma membrane.

964

\section{Supplementary movie $6 \& 7$.}

966 Plasmodesma Type II in Arabidopsis cultured cells.

967 The movies display $0.56 \mathrm{~nm}$ thick tomographic slices and a manual segmentation of the

968 data shown in Supplementary Fig.4. ER/desmotubule in pale blue, PM in orange and 969 tethering elements in red.

970

971 Supplementary Fig 8.

972 Classic TEM micrographies of representative Type I (a, b) and Type II (c, d) 973 plasmodesmata in gray levels and fake Fire coloring to highlight the presence or absence 974 of cytoplasmic sleeve.

975

976

977

978

979

980

981

982

983

984

985

986

987

988 


\section{Acknowledgements}

993 This work was supported by the grants by the Region Aquitaine (to E.M.B) and PEPS 994 (Initial Support for Exploratory Projects to E.M.B) and National Agency for Research 995 (Grant ANR-14-CE19-0006-01 to E.M.B). Electron and fluorescence microscopy analyses 996 were performed at the Bordeaux Imaging Centre (http://www.bic.ubordeaux2.fr). The 997 Region Aquitaine also supported the acquisition of the electron microscope (grant $\left.998 \quad \mathrm{n}^{\circ} 20111304007 \quad \mathrm{PFM}\right)$. We thank Yoselin Benitez-Alfonso for providing the 999 Arabidopsis PA-GFP lines and Sébastien Mongrand, Vincent Arondel, Yohann Boutté, 1000 Yvon Jaillais and Chris Hawes for critical review of the article prior to submission.

\section{Author contributions}

1003 Electron microscopy and associated-quantitative analyses were done by W.J.N. with the 1004 help of S.T. and L.B.. M.S.G. performed the cell-to-cell connectivity essays with the help of 1005 W.J.N and L.B. F.P.C. and L.B. provided technical support for the FRAP experiments and 1006 with image quantification and acquisition. All statistical analyses were run by W.J.N. and 1007 M.S.G. A.G. and M.F. performed the control tests for latrunculin and cytochalasin 1008 treatments in the roots. The manuscript was written by E.M.B. and W.J.N. with 1009 contributions of L.B., K.O. and J.T. Research was designed by E.M.B.

\section{Additional information}

1012 Correspondence and requests for materials should be addressed to W.J.N and E.M.B.

\section{Competing interests}

1015 The authors declare no competing financial interests. 

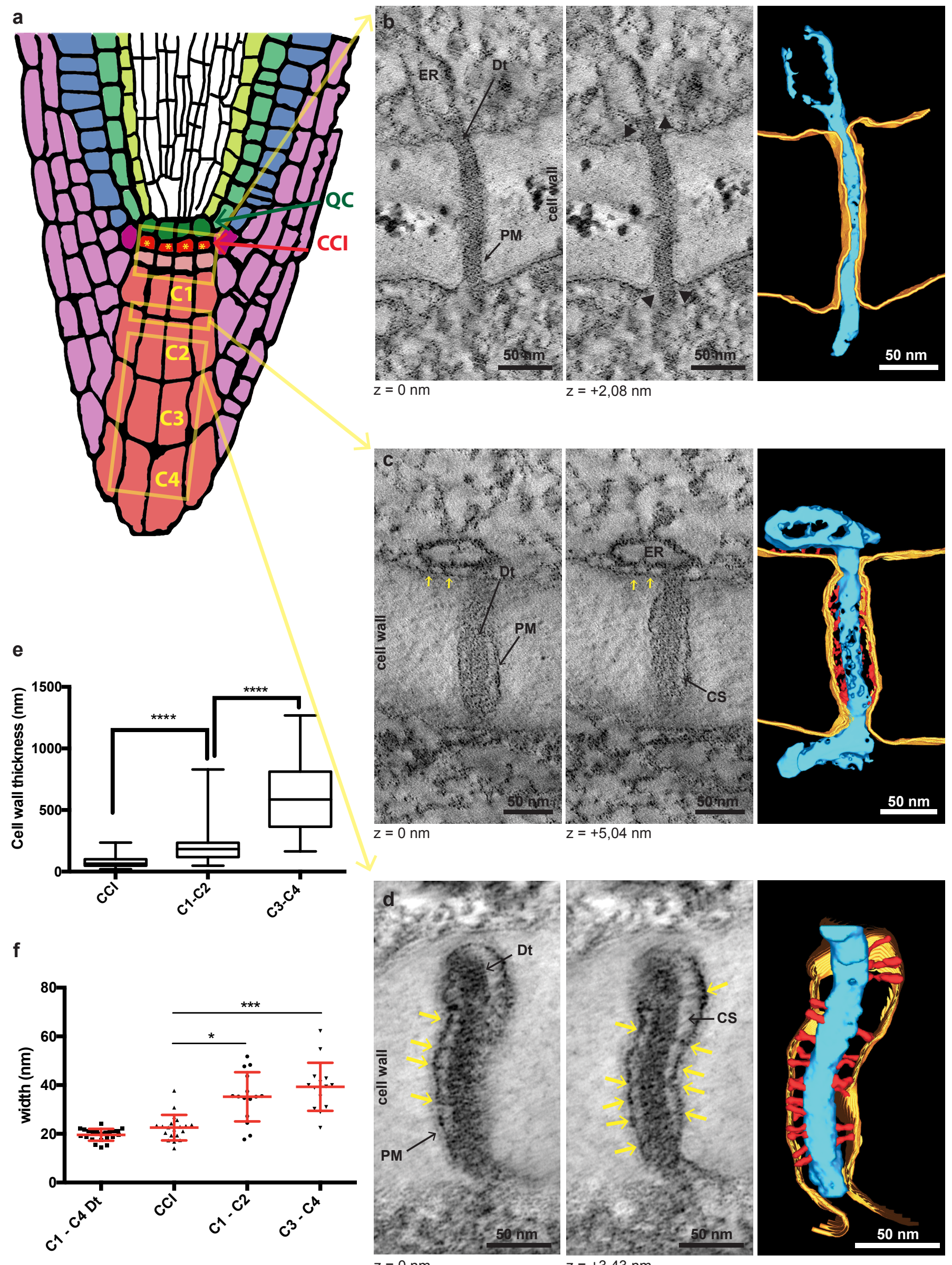
Figure 1. Plasmodesmata ER-PM contact site morphology evolves during tissue development in root tips.

(a) Schematic representation of Arabidopsis root tip cellular organisation, in which the colours depict cell lineage. Plasmodesmal ultrastructure was studied in the Columella tiers in light red (yellow boxes), from the $\mathrm{C} 1$ to $\mathrm{C} 4$ layer and in the $\mathrm{COL}$ cell initials in red ( $\mathrm{CCl}$, yellow asterisks). (b-d) Tomographic slices of representative plasmodesmata from the $\mathrm{CCl}$ (b), C1-C2 (c) and C3-C4 (d) interfaces, respectively $0.56,0.56$ and $0.49 \mathrm{~nm}$ thick, and their associated $3 \mathrm{D}$ segmentation highlighting the changes in ER-PM architecture within the pores during $\mathrm{COL}$ cell differentiation. In $\mathrm{CCl}$, appressed ER is seen entering the plasmodesmal pores (black arrowheads) and fills the entire canal (b). With tissue differentiation the cytoplasmic sleeve becomes gradually visible as the gap between the desmotubule and PM expands and become populated by spoke-like tethers (d, yellow arrows). In the $\mathrm{C} 1 / \mathrm{C} 2$ transition zone there is no clearly identifiable spoke-elements in the cytoplasmic sleeve, only amorphous material (c, red). (e) Difference in cell wall thickness in $\mathrm{CCl}$, C1-C2 and C3-C4 cells (Dunn's multiple comparison test ${ }^{* * *} \mathrm{P}<0.0001$ ). $\mathrm{n}=94$ for $\mathrm{CCl}, \mathrm{n}=27$ for $\mathrm{C} 1-\mathrm{C} 2$ and $\mathrm{n}=79$ for $\mathrm{C} 3-\mathrm{C} 4$. (f) Average diameter of plasmodesmata (PM-PM, inner leaflets) and desmotubules in $\mathrm{CCl}, \mathrm{C} 1-\mathrm{C} 2$ and C3-C4 cells (Dunn's multiple comparison test, $\left.{ }^{*} \mathrm{P}<0.05,{ }^{* * *} \mathrm{P}<0.001\right) . \mathrm{n}=20, \mathrm{n}=15$ and $\mathrm{n}=15$ plasmodesmata tomograms for $\mathrm{CCl}, \mathrm{C} 1-\mathrm{C} 2$ and $\mathrm{C} 3-\mathrm{C} 4$, respectively. $\mathrm{CCl}$ : columella cell initials; CS: cytoplasmic sleeve; Dt: desmotubule; ER: endoplasmic reticulum; $\mathrm{QC}$ : quiescent centre; PM: plasma membrane. 


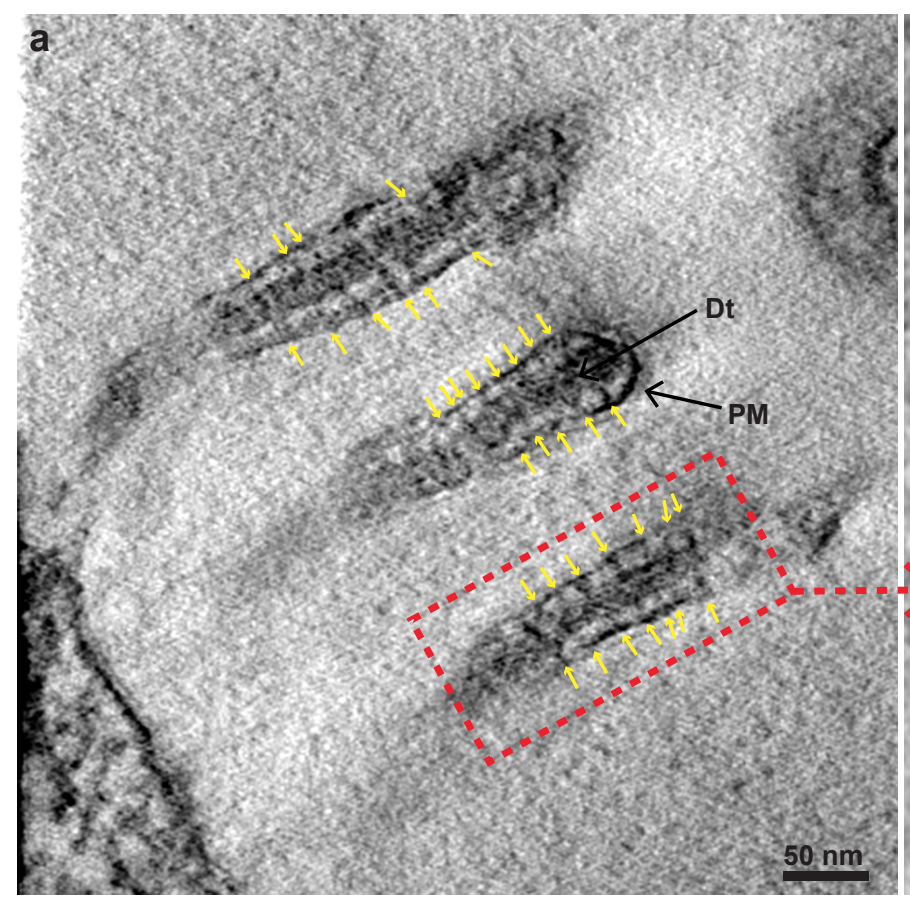

d

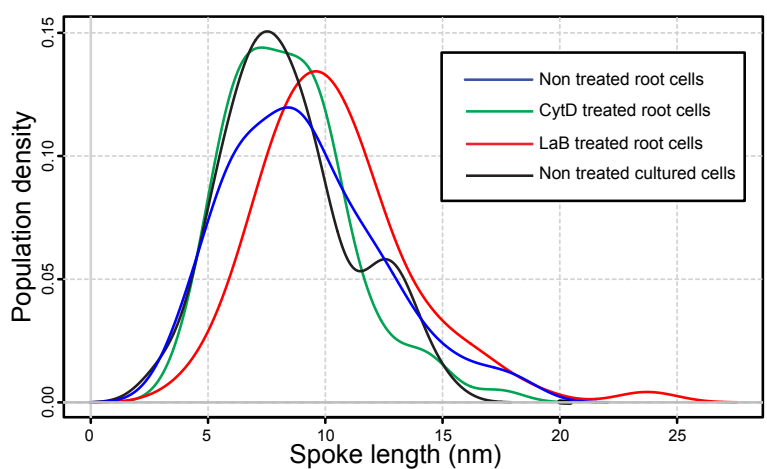

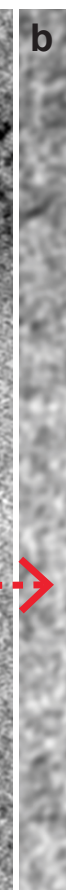

e

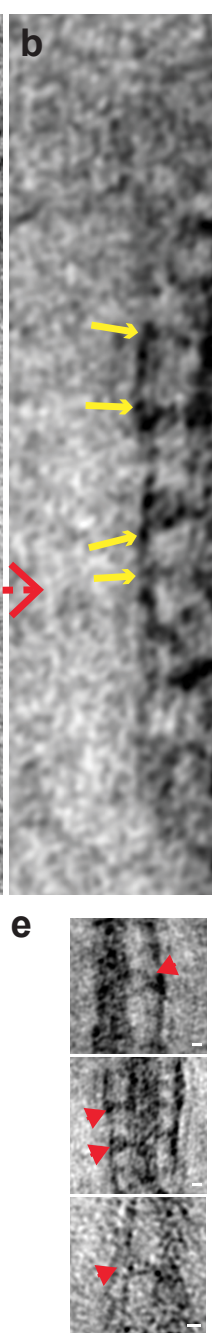

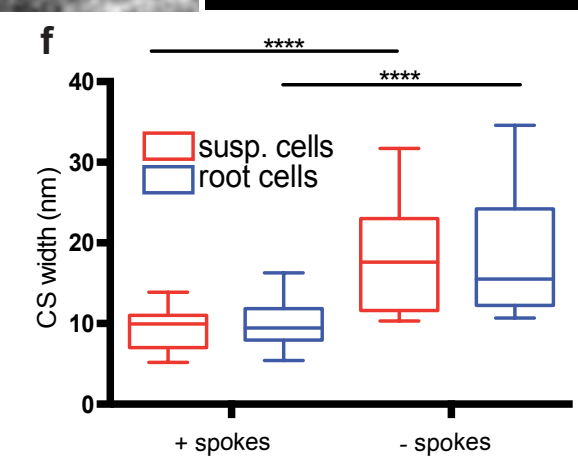

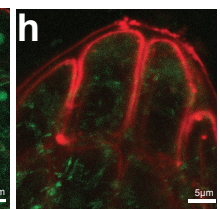

CytD

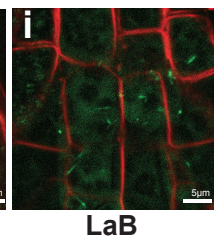

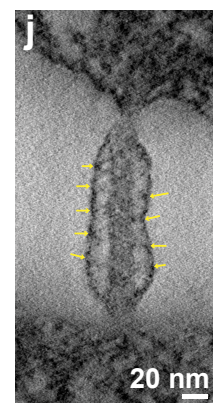

Cyt D
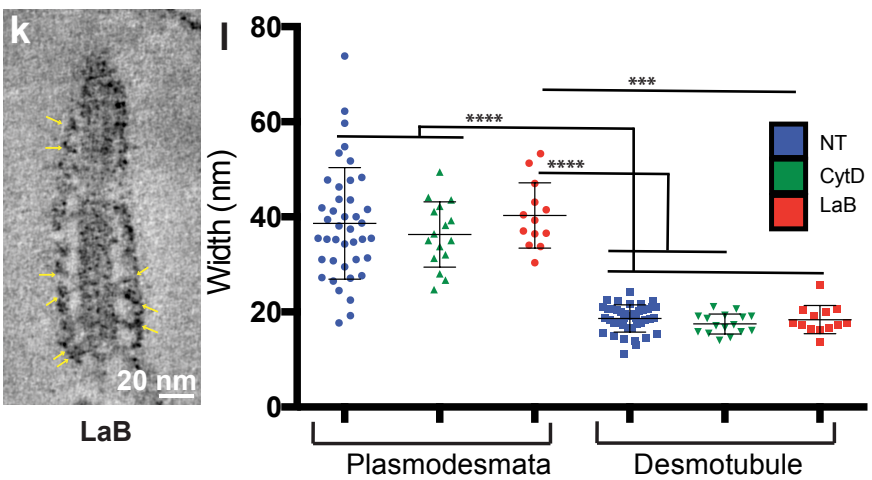

Plasmodesmata

Desmotubule

$20 \mathrm{~nm}$

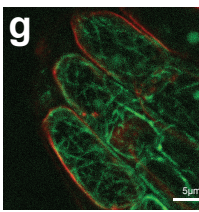

Control

LaB

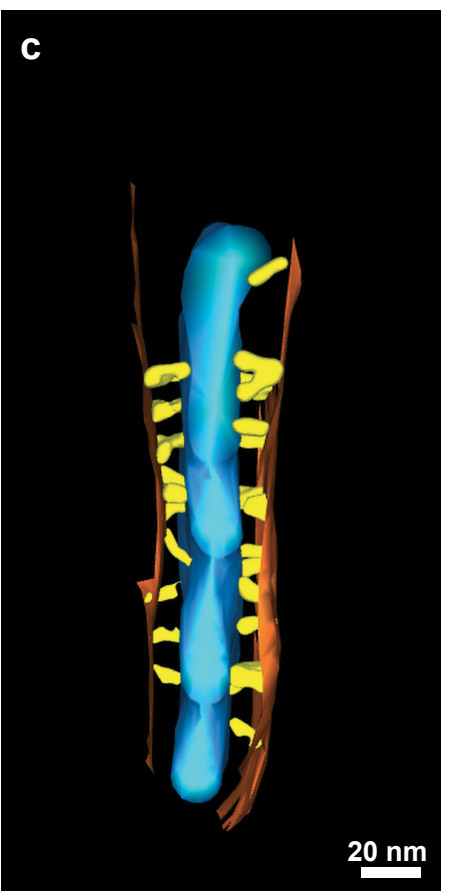


Figure 2. The spoke-like tethering elements of Type II plasmodesmata correlate with ER-PM spacing and are not sensitive to F-actin polymerization inhibitor drugs

(a-c) $1.24 \mathrm{~nm}$ thick tomographic slices depicting the typical and regular arrangement of the spoke-like tethering elements (yellow arrows) in Type II plasmodesmata, in the root columella (C2-C4). (b) Close-up view of the plasmodesma squared in (a). (c) Manual segmentation of (b). (d) Density representation of the spoke length, measured in COL cells and cultured Arabidopsis cells (non-treated, Latrunculin B, $\mathrm{LaB}$ and Cytochalasin D, CytD treated for roots and non treated for cultured cells). In either condition, there is a peak density for spokes of about $9 \mathrm{~nm}$ in length. (e) Three close-up views of $V / Y$ shaped spokes (red arrowheads) oriented towards the PM (top; middle) and the desmotubule (bottom). Scale bars $=5 \mathrm{~nm}$. (f) Plot representation of the cytoplasmic sleeve gaps (desmotubule - PM distance) measured in subsections of transitioning or Type II pores with or without spokes. Both in roots and cultured cells the presence of spokes stabilizes the width of the intermembrane gap and keeps it at an average of $9.6 \pm 2.44 \mathrm{~nm}$ whereas without the spokes this gap is $18.38 \pm 7.26 \mathrm{~nm}$ (Mann-Whitney two tailed test, $\left.{ }^{* * * *} P<0.0001\right) n=$ 31 and $n=65$ measurements in cell and root plasmodesmata respectively.

(g-I) Type II plasmodesmata in $\mathrm{COL}$ cells (C2-C4) are not altered by F-actinpolymerization inhibitor drugs. (g-i) Maximum projections of confocal stacks taken in columellas of Arabidopsis marker lines fimbrin actin binding domain 35S::GFPfABD2-GFP. In control conditions (g), cells show a dense, reticulated actin network, while after $1 \mathrm{~h} 30$ hour treatment with $50 \mu \mathrm{M}$ Cytochalasin $\mathrm{D}(\mathrm{CytD}, \mathbf{h})$ or Latrunculin $B$ $(\mathrm{LaB}, \mathrm{i})$, the F-actin network is heavily altered. (j-k) $0.56 \mathrm{~nm}$ thick tomographic slices of plasmodesmata acquired in the columella of CytD (j) and LaB (k)-treated roots, showing that the spokes are still present. (I) The diameter (PM-PM; innerleaflets) of the pores and the desmotubule width remain unchanged both treatment (Dunn's multiple comparison test, $\left.{ }^{* * * *} \mathrm{P}<0.0001,{ }^{* * *} \mathrm{P}<0.001\right) \mathrm{n}=41$ plasmodesmata tomograms for non-treated (NT) condition, $n=13$ for LaB and $n=16$ for CytD treated condition. Dt: desmotubule ; PM : plasma membrane. 

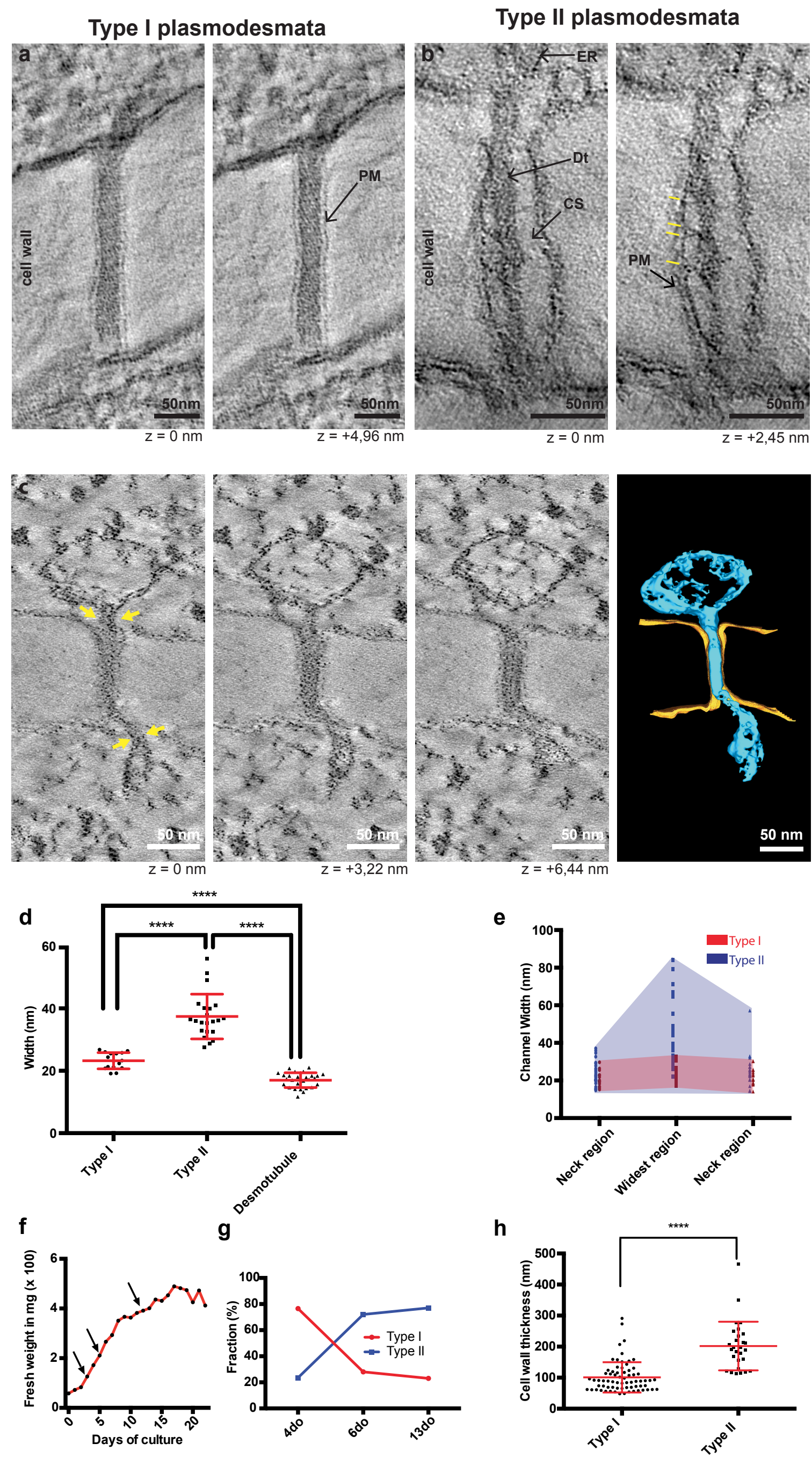
Figure 3. Very tight ER-PM contact in post-cytokinesis plasmodesmata

(a-b) Consecutive tomographic slices, of respectively $1.24 \mathrm{~nm}$ and $0.49 \mathrm{~nm}$ in thickness, of a Type I plasmodesma (a) and Type II plasmodesma (b, spokes are indicated by yellow dashes) in Arabidopsis cultured cells. (c) Type I plasmodesmata are traversed by the ER which becomes appressed just before entering the pores (yellow arrows). Three $0.56 \mathrm{~nm}$ thick tomographic slices and the corresponding 3D segmentation. (d) Plasmodesmata diameter (PM-PM; inner leaflets) of Type I (23 \pm $2.6 \mathrm{~nm})$ and Type II $(37 \pm 7.2 \mathrm{~nm})$, and the desmotubule measured in Type II plasmodesmata $(17 \pm 2.4 \mathrm{~nm}) . \mathrm{n}=17$ and 22 tomograms for Type I and Type II plasmodesmata, respectively, and $n=22$ for desmotubule measurements $\left({ }^{* * *} \mathrm{P}<\right.$ 0.0001 by Mann-Whitney test). (e) plasmodesmata width at different points along the pores in Type I and II. Measurements (PM-PM; inner leaflets) were taken at the extremities and largest part of the channels. Type I plasmodesmata have a remarkably constant diameter compared to Type II. $n=17$ and $n=22$ tomograms for Type I and Type II plasmodesmata, respectively. (f) Growth curve of Arabidopsis liquid cultured cells. Black arrows indicate the cell culture ages used for this study (four-, six- and thirteen-day-old). (g) Quantification of the relative proportion of Type I and II plasmodesmata in Arabidopsis cultured cells at four, six and thirteen days ( $\mathrm{n}=$ 111, 89 and 22 screened plasmodesmata for four, six and thirteen days old cells). (h) Average cell wall thickness in relation to plasmodesmata Type. In four-day-old cultured cells, Type I plasmodesmata are abundant in thin young cell walls whereas Type II plasmodesmata are preferentially associated with thicker, older walls. $n=69$ and 28 for Type I and II plamodesmata, respectively ${ }^{* * * *} \mathrm{P}<0.0001$ by MannWhitney test). For (g) and (f), plasmodesmata screening was done on $90 \mathrm{~nm}$ thick sections by TEM (see M\&M for details). CS: cytoplasmic sleeve; Do: days of culture; Dt: desmotubule; ER: endoplasmic reticulum; PM: plasma membrane. 


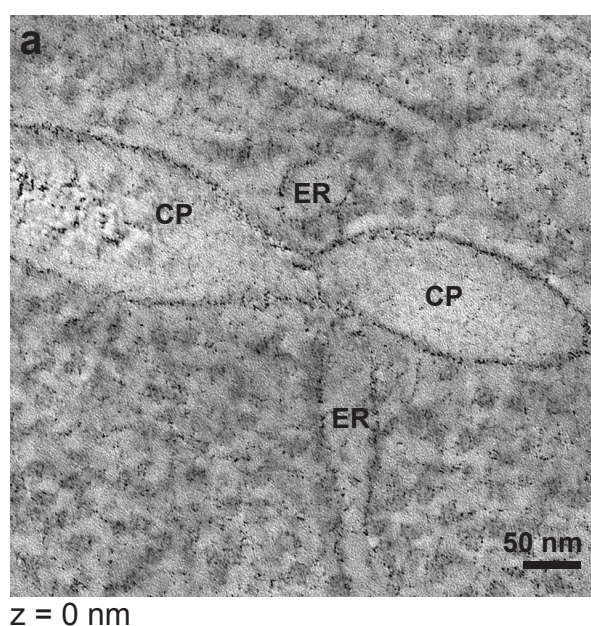

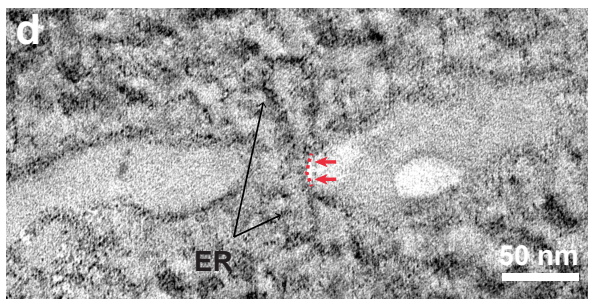
$\mathrm{z}=0 \mathrm{~nm}$

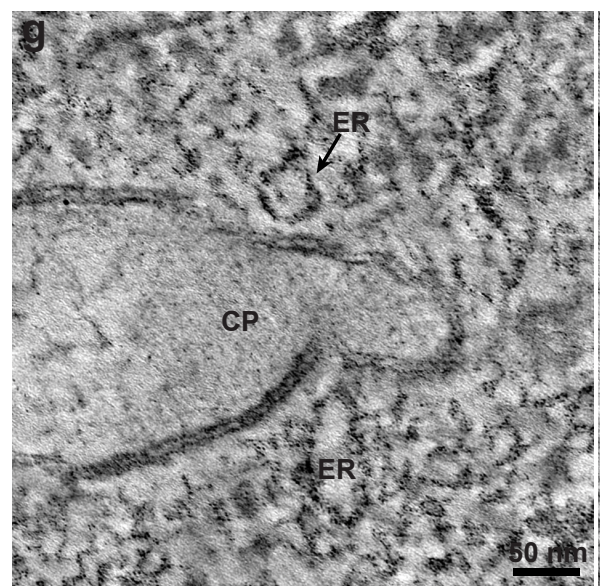

$\mathrm{z}=0 \mathrm{~nm}$

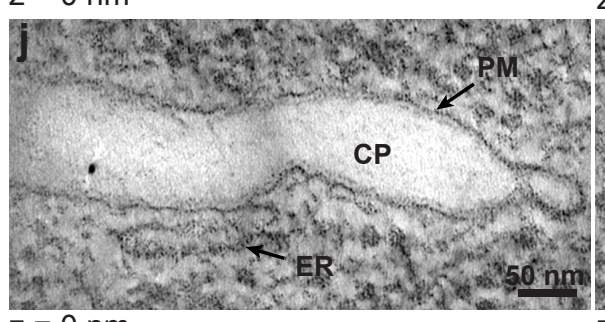

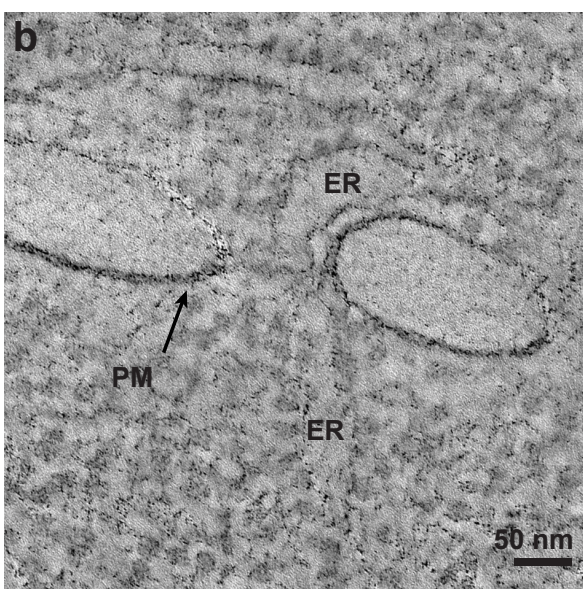

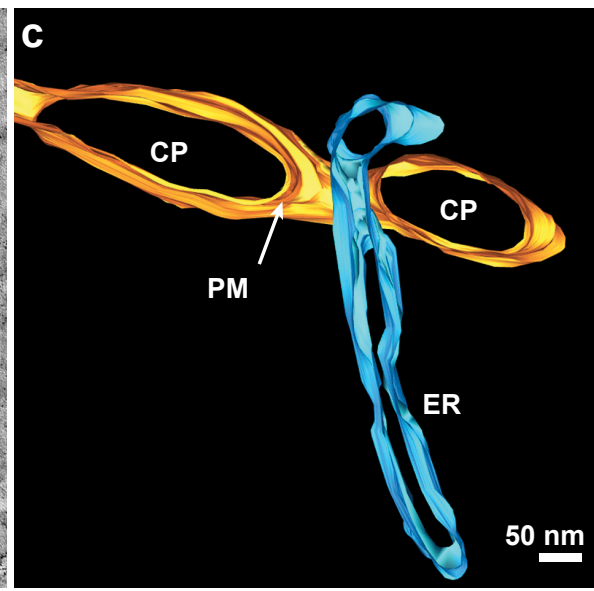

$$
z=+29,44 \mathrm{~nm}
$$

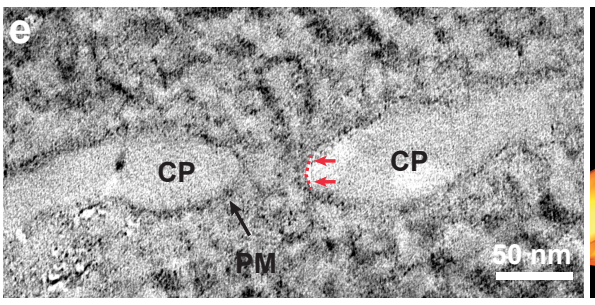
$z=+4,14 \mathrm{~nm}$

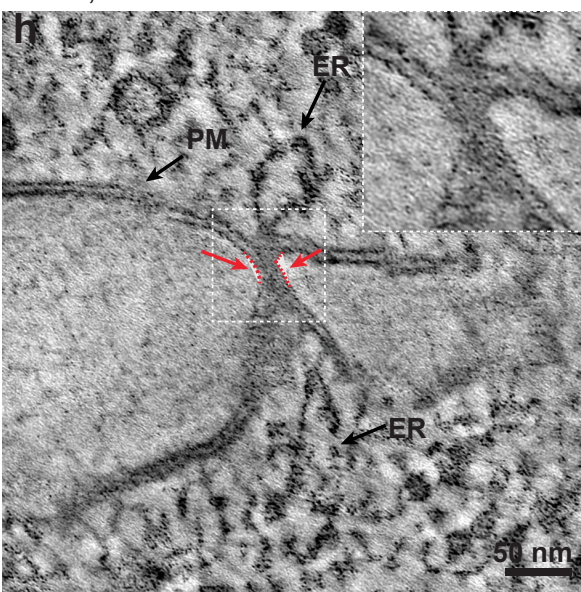

$$
\mathrm{z}=+16,24 \mathrm{~nm}
$$
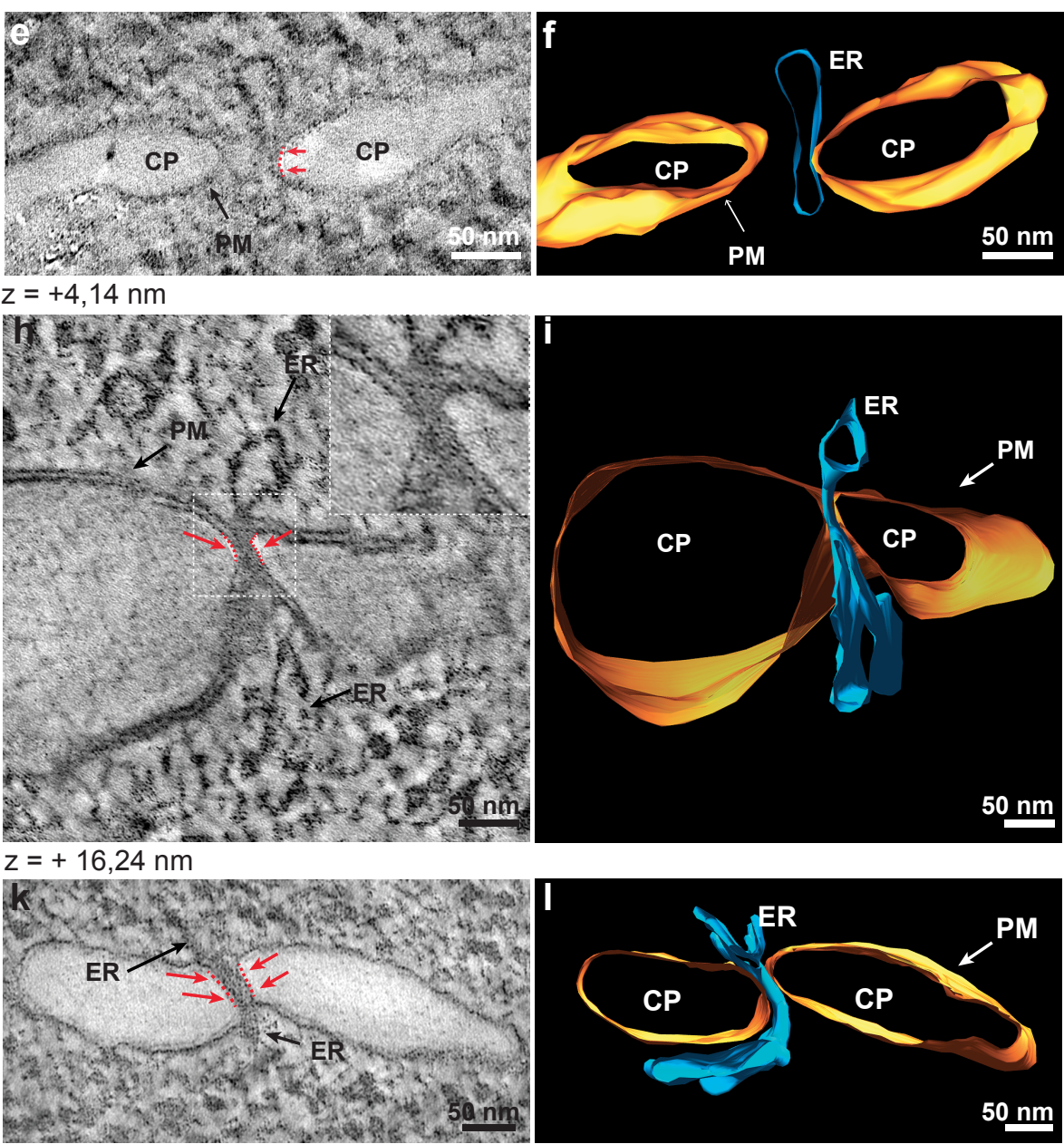
Figure 4. Very tight ER-PM contacts are established during cell plate formation. (a-f) $0.46 \mathrm{~nm}$ thick tomographic slices, and the associated segmentation show nonappressed ER strands trapped by the fenestrated cell plate, establishing very tight contacts ( $d-f$, red arrows and dashed line) at very early stages of plasmodesmata initiation. (g-l) $0.56 \mathrm{~nm}$ thick $(\mathrm{g}, \mathrm{h})$ and $0.36 \mathrm{~nm}$ thick $(\mathbf{j}, \mathbf{k})$ tomographic slices depicting the establishment of very tight ER-PM contacts occurring on one end of the forming plasmodesma ( $g-i$, red arrows and dashed line) and along its entire length ( $j-$ I, red arrows and dashed line). CP: cell plate; ER: endoplasmic reticulum; PM: plasma membrane. 
a
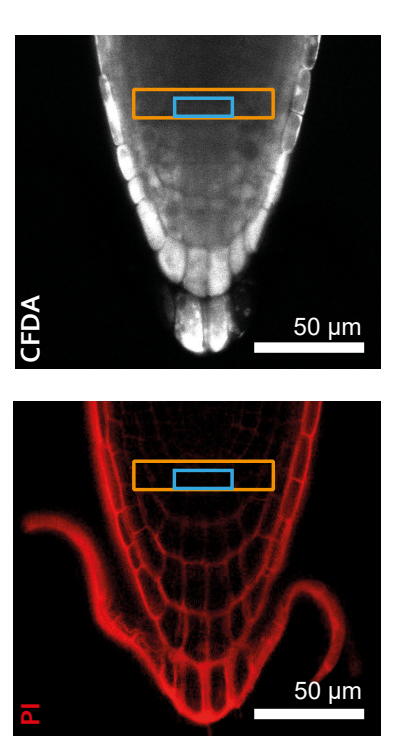

d

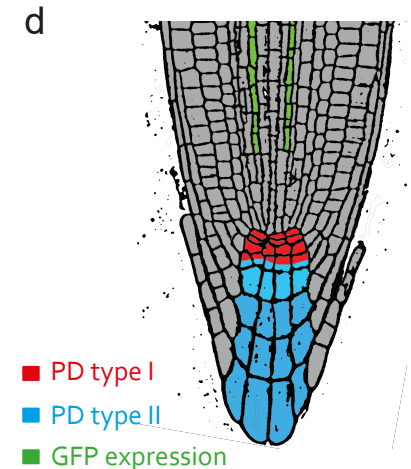

e

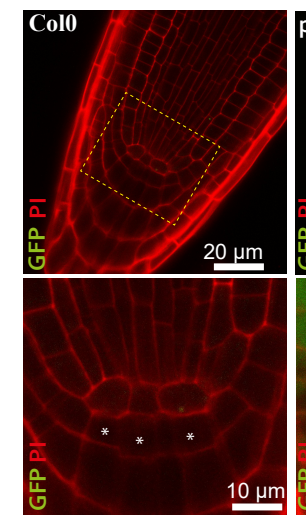

g

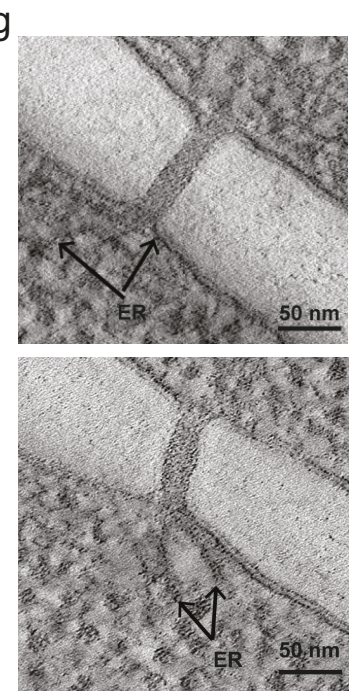

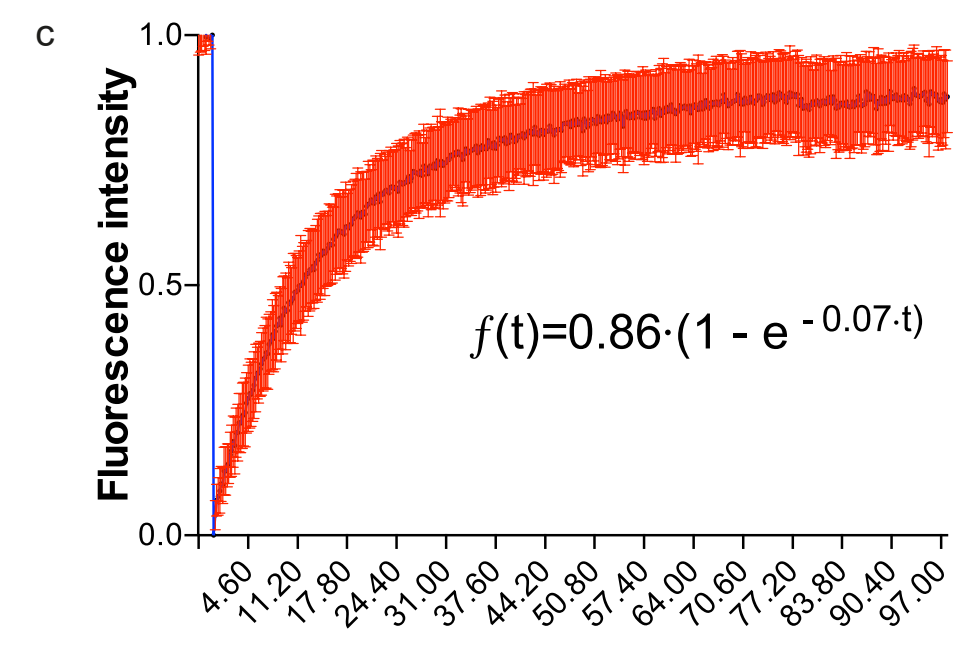

Time (s)

b

a

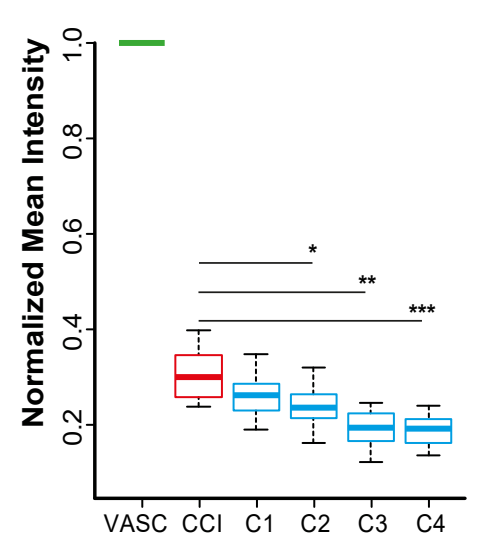

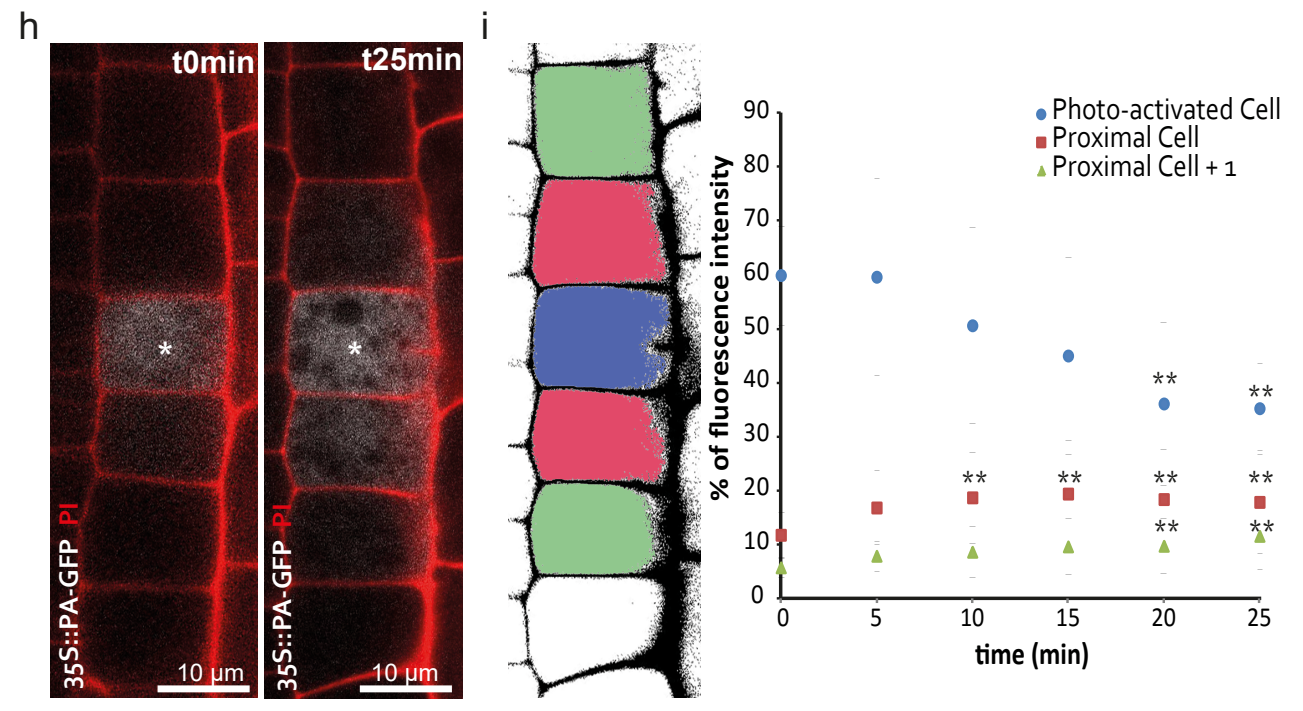




\section{Figure 5. Molecular trafficking through Type I plasmodesmata}

(a-c) Plasmodesmata permeability at the $\mathrm{CCl} / \mathrm{C} 1$ interface monitored by FRAP and CDFA (a) Col. O root tip co-stained with CFDA and Propidium lodide. Orange and blue $(\mathrm{CCl})$ boxes indicate region that were photobleached and where fluorescence intensity was monitored, respectively. (b) Representative kymograph of $\mathrm{CCl}$ region (blue box in a). Fire LUT was applied to enhance visualization of the photobleaching and recovery. (c) Mean recovery curve with error bars indicating standard deviation (3 independent experiments; 10 successful FRAPs in 10 individual roots), showing rapid recovery of CFDA within the $\mathrm{CCl}$. The one-phase exponential association curve fit $\left(R^{2}=0.86\right)$ calculated a half-time recovery of 9.7 seconds, a $\mathrm{K}$ constant of $0.07 \mathrm{~s}^{-1}$ and a Ymax of 0.86

(d-f) Non-targeted diffusion of free GFP in the COL cells using pSUC2::GFP lines. (d) Cartoon of Arabidopsis pSUC2::GFP root. Green cells represent the companion cells where the GFP is expressed. The presence of GFP in other parts of the root is due to diffusion through plasmodesmata. Red and blue colours show cell interfaces harbouring Type I or Type II plasmodesmata, respectively. (e) Confocal slices through Col 0 (left panels) and pSUC2::GFP (right panels) root tips exhibiting GFP signal (green) in the columella. Close up view (yellow boxed regions in upper panels) in the meristematic region show the $\mathrm{CCl}$ contours in the propidium iodide channel (white stars in the two bottom panels) reveal the CCl cells, right below the periblem layer containing the quiescent centre. GFP signal is visible in the meristematic area of pSUC2::GFP lines, in contrast to the absence of fluorescence in the Col.0 root tip. (f) GFP fluorescent quantification in the pSUC2::GFP lines in $\mathrm{CCl}$, and $\mathrm{C} 1-\mathrm{C} 4 \mathrm{COL}$ layers (background was subtracted against Col-0 roots). Intensities are normalized within a given root relative to the intensity in the vascular system (set to 1$)$. $(n=15$ pSUC2::GFP roots and $n=10$ Col.0 roots in 3 independent experiments). Wilcoxon test was used to compare each cell type to the $\mathrm{CCl}$ cells. ${ }^{*} \mathrm{P}<0.05$, ${ }^{* *} \mathrm{P}<0.01$, ${ }^{* * *}$ $P<0.001$.

(g-i) Photoactivated (PA)-GFP diffusion through Type I plasmodesmata. (g) Two 0.56 $\mathrm{nm}$ thick tomographic slices of Type I plasmodesmata in transversal walls of epidermal cells in the root meristematic zone. (h) Confocal slices showing PA-GFP signal in photoactivated cell (t0; white asterisk) and reaching the neighbouring cells after t25 minutes. Right panel represents a color-coded cartoon. (i) Fluorescence was quantified in the photo-activated (blue) and the adjacent ( $n$ proximal, in red and $n+1$ in green) cells. PA-GFP fluorescence in activated cells consistently showed a decrease of intensity over time whereas neighbouring cells $(n, n+1)$ showed a concomitant increase in fluorescence. ( $n=15$ roots; 5 independent experiments). Two-tailed Wilcoxon test was used to compare the fluorescence intensity in a given cell over time. The subsequent times points after photoactivation were always tested with to as the reference, for a given cell. ${ }^{* *} \mathrm{P}<0.01$. CCl: columella cell initial; CFDA: carboxyfluorescein diacetate; COL: columella; FRAP: Fluorescence recovery after photobleaching; LUT: look up table. 